\title{
The Range of a Contractive Projection in $L_{p}(H)$
}

\author{
Yves RAYNAUD \\ Institut de Mathématiques de Jussieu (CNRS), \\ Projet Analyse Fonctionnelle (Case 186), \\ Université Paris-VI, \\ 4, place Jussieu, \\ 75252 Paris Cedex 05, France. \\ yr@ccr.jussieu.fr
}

Recibido: 21 de Noviembre de 2003

Aceptado: 10 de Mayo de 2004

\begin{abstract}
We show that the range of a contractive projection on a Lebesgue-Bochner space of Hilbert valued functions $L_{p}(H)$ is isometric to a $\ell_{p}$-direct sum of Hilbertvalued $L_{p}$-spaces. We explicit the structure of contractive projections. As a consequence for every $1<p<\infty$ the class $\mathcal{C}_{p}$ of $\ell_{p}$-direct sums of Hilbertvalued $L_{p}$-spaces is axiomatizable (in the class of all Banach spaces).

Key words: Contractive projections, Vector-valued $L_{p}$-spaces.

2000 Mathematics Subject Classification: Primary: 46B04, 46E40. Secondary: 46M07.
\end{abstract}

\section{Introduction}

It was a remarkable achievement in the isometric theory of Banach spaces of the years 1960's to characterize the contractive linear projections of Lebesgue $L_{p}$ spaces $(p \neq 2)$. In the case of $L_{p}$ spaces of a probability space it was done by Douglas [4] in the case $p=1$ and Andô [1] in the case $1<p<\infty, p \neq 2$. They showed that the range of such a contractive projection is itself isometric to a $L_{p}$ space (for the same $p$, but a different measure space); if moreover the projection is positive then its range is a sublattice of the initial $L_{p}$ space and is lattice isomorphically isometric to a $L_{p}$ space. This was extended to the non-sigma-finite measure space setting by Tzafriri $([17])$. In the case of a probability space, the structure of contractive projections is 
elucidated by Douglas-Andô works: a general contractive projection $P$ on $L_{p}(\Omega, \Sigma, \mu)$ has the form

$$
P=M_{\varepsilon} \widehat{P} M_{\varepsilon}^{-1}+V
$$

where $M_{\varepsilon}$ is the multiplication operator by a function $\varepsilon$ with $|\varepsilon|=\mathbf{1}, \widehat{P}$ is a positive contractive projection, and $V=0$ if $p>1$, while if $p=1$, then $V$ is a contraction from $L_{1}$ into the range $R(P)$ of $P$ which vanishes on the band generated by $R(P)$. Moreover $\widehat{P}$ is a weighted conditional expectation, i.e. there exist a sub-sigma algebra $\mathcal{B}$, an element $B \in \mathcal{B}$ and a nonnegative function $w \in L^{p}$ such that $\mathbb{E}\left(w^{p} \mid \mathcal{B}\right)=\mathbf{1}$ and

$$
\widehat{P} f=w \mathbb{E}\left(\mathbf{1}_{B} f \cdot w^{p-1} \mid \mathcal{B}\right)
$$

for every $f \in L_{p}$ (in particular if $P \mathbf{1}=\mathbf{1}$ then $P$ is a conditional expectation). This last formula can also be written

$$
\widehat{P} f=w \mathbb{E}_{\nu}\left(\mathbf{1}_{B} f w^{-1} \mid \mathcal{B}\right)
$$

where $\mathbb{E}_{\nu}$ is the conditional expectation relative to the measure $\nu=w^{p} \cdot \mu$. If we denote by $S$ the isometric isomorphism $f \mapsto w \cdot f$ of $L_{p}(\Omega, \Sigma, \nu)$ onto $L_{p}(\Omega, \Sigma, \nu)$ and by $M_{B}$ the multiplication operator by the indicator function $\mathbf{1}_{B}$, we have:

$$
\widehat{P}=S M_{B} \mathbb{E}_{\nu}(\mid \mathcal{B}) S^{-1} .
$$

The structure of contractive projections in the non-sigma finite case was treated by Bernau and Lacey ([3]); their main result can be rephrased in saying that if we assume (as we may) that the measure space $(\Omega, \Sigma, \mu)$ is localizable ([7]) then formulas (1) and (2) are still valid; now $w$ is some $\Sigma$-measurable positive function, $\nu=w^{p} \cdot \mu$ and $\mathcal{B}$ is some semi-finite sigma-subalgebra of $\Sigma$.

The task of extending these results to various classical spaces was considered by numerous authors; see the recent survey paper [15] and the references inside. Here we are more specifically interested in the case of vector-valued Lebesgue $L_{p}$ spaces, in particular mixed norm spaces $L_{p}\left(L_{q}\right)$. Since the survey paper [5] on this specific subject, several partial results appeared. In particular B. Randrianantoanina ([14]) succeeded in solving thoroughly the complex sequential case $\ell_{p}\left(\ell_{q}\right)$ using hermitian operator techniques introduced in the subject by Kalton and Wood. More recently the case of finite dimensional real Banach spaces with $C^{2}$ norm was considered by the authors of [12]; under some additional conditions on the dual norm (in particular it is assumed to be $C^{2}$ on the complementary set of the coordinate hyperplanes associated to a distinguished basis) the contractively complemented subspaces are shown to be necessarily generated by a block-basis of the given basis. This can be applied in particular to the real spaces $\ell_{p}^{n}\left(\ell_{q}^{m}\right)$, when $2<p, q<\infty$ (or by duality when $1<p, q<2$ ), obtaining the same description of their contractively complemented subspaces as in the complex case [16].

In the present paper we examine the case of Lebesgue spaces of Hilbert valued functions $L_{p}(H)$; this is done in the most general case (without any assumption of 
sigma-finiteness of $L_{p}$-space or separability of the Hilbert space; in fact we have in mind some applications to the ultrapowers of such spaces, which are neither separable nor sigma-finite). It turns out that the range of a contractive projection is a $\ell_{p}$-direct sum of spaces of the type $L_{p}(H)$. More precisely:

Theorem 0.1. Let $1 \leq p<\infty, p \neq 2 ; H$ be a Hilbert space and $L_{p}=L_{p}(\Omega, \Sigma, \mu)$. The range of every contractive projection $P: L_{p}(H) \rightarrow L_{p}(H)$ is isometric to a $\ell_{p}$-direct sum of Hilbert-valued $L_{p}$-spaces, i.e.

$$
R(P) \approx_{1}\left(\bigoplus_{i \in I} L_{p}\left(\Omega_{i}, \mathcal{B}_{i}, \mu_{i} ; H_{i}\right)\right)_{\ell_{p}}
$$

where $\left(\Omega_{i}\right)_{i}$ is a family of pairwise almost disjoint members of $\Sigma$, each $\mathcal{B}_{i}$ is a subsigma-algebra of the trace $\Sigma_{i}$ of $\Sigma$ on $\Omega_{i} ; \mu_{i}$ is the trace on $\Omega_{i}$ of the measure $\mu$; and the Hilbert spaces $H_{i}$ have Hilbertian dimension not greater than the Hilbertian dimension of $H$.

Conversely a $\ell_{p}$-sum $\left(\bigoplus_{i \in I} L_{p}\left(\Omega_{i}, \Sigma_{i}, \mu_{i} ; H_{i}\right)\right)_{\ell_{p}}$ embeds isometrically into $L_{p}(H)$, where $L_{p}=\left(\bigoplus_{i \in I} L_{p}\left(\Omega_{i}, \mathcal{B}_{i}, \mu_{i}\right)\right)_{\ell_{p}}$ and $H=\left(\bigoplus_{i \in I} H_{i}\right)_{\ell_{2}}$. Hence a contractively complemented subspace of a $\ell_{p}$-direct sum of Hilbert-valued $L_{p}$-spaces is still a $\ell_{p^{-}}$ direct sum of Hilbert-valued $L_{p}$-spaces. In other words:

Corollary 0.2. The class $\mathcal{C}_{p}$ of $\ell_{p}$-direct sums of Hilbert-valued $L_{p}$-spaces is stable under contractive projections.

The structure of the contractive projection $P$ can be easily explained in the case where the space $H$ is separable (the non-separable case is analogous and will be described in Section 5). Recall that given two Banach spaces $X, Y$, a family of operators $T_{\omega}: X \rightarrow Y$ is said to be strong-operator $\Sigma$-measurable if for every $x \in X$, the map $\omega \mapsto T_{\omega} x$ is $\Sigma$-measurable as a map $\Omega \rightarrow Y$. If moreover Ess $\sup _{\omega}\left\|T_{\omega}\right\|<\infty$, such a measurable family induces a bounded linear map $T$ from $L_{p}(\Omega, \Sigma, \mu ; X)$ into $L_{p}(\Omega, \Sigma, \mu ; Y)$ by the equation:

$$
(T f)(\omega)=T_{\omega}\left(f_{\omega}\right)
$$

Theorem 0.3. Under the conditions of Thm. 0.1, if moreover $H$ is separable, then

$$
P=\sum_{i \in I} S_{i}\left(\widetilde{P}_{i} \otimes \operatorname{Id}_{H_{i}}\right) S_{i}^{\sharp} M_{\Omega_{i}}+V
$$

where $\widetilde{P}_{i}$ is a positive contractive projection in $L_{p}\left(\Omega_{i}, \Sigma_{i}, \mu_{i}\right) ; S_{i}$ is an isometric embedding of $L_{p}\left(\Omega_{i}, \Sigma_{i}, \mu_{i} ; H_{i}\right)$ into $L_{p}\left(\Omega_{i}, \Sigma_{i}, \mu_{i} ; H\right)$ associated with a (strong-operator)measurable family $\left(S_{i, \omega}\right)_{\omega \in \Omega_{i}}$ of isometric embeddings $H_{i} \rightarrow H$, while $S_{i}^{\#}$ is associated with the adjoint family $\left(S_{i, \omega}^{*}\right)_{\omega \in \Omega_{i}}$ of projections $H \rightarrow H_{i} ; M_{\Omega_{i}}: L_{p}(\Omega ; H) \rightarrow$ $L_{p}\left(\Omega_{i} ; H_{i}\right)$ is the multiplication operator by the indicator function $\mathbf{1}_{\Omega_{i}}$; and $V=0$ if $p>1$, while if $p=1$ then $V$ is a contraction of $L_{1}(\Omega, \Sigma, \mu ; H)$ vanishing on every $L_{1}\left(\Omega_{i}, \Sigma_{i}, \mu_{i} ; H\right)$ and taking values in the range of $P$. 
Let us present shortly an application of the Thm. 0.1 which was in fact our main motivation for starting this study. If $X, Y$ are Banach spaces, we say that $X$ is an ultraroot of $Y$ if $Y$ is isometric to some ultrapower of $X$. Recall that a Banach space $X$ embeds canonically isometrically in every of its ultrapowers $X_{\mathcal{U}}$, and that if $X$ is reflexive, then this canonical image is contractively complemented in $X_{\mathcal{U}}$. As a consequence of Thm. 0.1 we see that every ultraroot of a $L_{p}(H)$ space, $p>1$ is a member of $\mathcal{C}_{p}$. By Cor. 0.2 the same is true for ultraroots of members of $\mathcal{C}_{p}$. On the other hand it was proved in [13] that every ultraproduct of $L_{p}(H)$ spaces is isometric to a $\ell_{p}$-direct sum of Hilbert-valued $L_{p}$-spaces. More generally every ultraproduct of members of $\mathcal{C}_{p}$ is itself isometric to a member of $\mathcal{C}_{p}$. Hence we obtain:

Corollary 0.4. For every $1<p<\infty$ the class $\mathcal{C}_{p}$ of $\ell_{p}$-direct sums of Hilbert-valued $L_{p}$-spaces is stable under ultraproducts and ultraroots.

In other words the class $\mathcal{C}_{p}$ is axiomatizable in the sense of Henson-Iovino [9] in their language of normed spaces structures (see [9], Thm. 13.8).

The paper is organized as follows: after a section devoted to definitions, notations and a general result on orthogonally complemented subspaces of $L_{p}(H)$, we have two sections of preliminary results distinguishing the case $p=1$ (Section 2) from the case $p>1$ (Section 3). In these sections it is proved that if $f$ belongs to the range of a contractive projection $P$, then the whole subspace $Z_{f}:=\overline{L_{\infty}(\Omega) \cdot f}$ is preserved by $P$ (i.e. $P Z_{f} \subset Z_{f}$ ) which suggests clearly a possible reduction to the scalar case. It is also proved that the "orthogonal projection" onto $Z_{f}$ preserves the range of $P$. This allows to find an "orthogonal system" in $R(P)$ which generates $Z_{P}:=\overline{L_{\infty}(\Sigma) \cdot R(P)}$ over $L_{\infty}(\Sigma)$ which will furnish the orthogonal bases of the Hilbert spaces $H_{i}$ of Thm. 0.1. Section 4 is devoted to the proof of Thm. 0.1; a key point consists in proving that the different subalgebras of $\Sigma$ given by the scalar theorem (applied to each $Z_{f}$ ) are induced by the same sigma-subalgebra $\mathcal{F}$ of $\Sigma$. Finally Thm 0.3 is proved in Section 5 (in a more general version not requiring separability).

\section{General preliminaries}

\subsection{Definitions and notations}

Let $1 \leq p<\infty, H$ be an Hilbert space and $(\Omega, \Sigma, \mu)$ be a measure space. In the following we denote (when there is no ambiguity) by $L_{p}(H)$ the Lebesgue-Bochner space $L_{p}(\Omega, \Sigma, \mu ; H)$ of classes of $H$-valued $p$-integrable functions (for $\mu$-a.e. equality). Similarly $L_{\infty}(H)$ will be the space of classes of Bochner measurable, essentially bounded $H$-valued functions. These spaces can be defined directly from the Banach lattices $L_{p}$ (resp. $L_{\infty}$ ) and the Hilbert space $H$, but we adopt the functional point of view for the simplicity of the exposition. In the case where $(\Omega, \Sigma, \mu)$ is not sigmafinite, it is preferable to suppose that this measure space is localizable: the measure $\mu$ is semifinite (every set in $\Sigma$ of positive measure contains a further one of positive and 
finite measure) and $L_{\infty}(\Omega, \Sigma, \mu)$ is order complete. In particular every family $\left(A_{i}\right)_{i \in I}$ in $\Sigma$ has a supremum $A$, denoted by $\bigvee_{i \in I} A_{i}$. The set $A$ is defined (up to a $\mu$-null set) by the conditions:

\section{$A \supset A_{i}$ for every $i \in I$,}

If $B \in \Sigma$ and $B \dot{\supset} A_{i}$ for every $i \in I$ then $B \dot{\supset} A$,

where $B \dot{\supset} A$ means $\mu(A \backslash B)=0$ (define similarly $A \dot{\subset} B$ and $A \doteq B$ ). We say that $B, C$ are almost disjoint if $A \cap B \doteq \emptyset$.

To every $f \in L_{p}(H)$ we associate its "random norm" $N(f) \in L_{p}^{+}$defined by $N(f)(\omega)=\|f(\omega)\|_{H}$, its vectorial function support $\mathbf{V S}(f)=\operatorname{Supp}(N(f))$ and its "random direction", i.e. the element $u_{f}$ of $L_{\infty}(H)$ defined by $u_{f}(\omega)=\frac{f(\omega)}{N(f)(\omega)}$ if $\omega \in \mathbf{V S}(f),=0$ if $\omega \notin \mathbf{V S}(f)$. If $M \subset L_{p}(H)$ we set $\mathbf{V S}(M)=\bigvee\{\mathbf{V S}(f) \mid f \in M\}$. If $f \in L_{p}(H), g \in L_{q}(H)$ we define their random scalar product $\langle\langle f, g\rangle\rangle \in L_{r}$ (where $\left.\frac{1}{r}=\frac{1}{p}+\frac{1}{q}\right)$ by $\langle\langle f \mid g\rangle\rangle(\omega)=\langle f(\omega) \mid g(\omega)\rangle_{H}$, where $\langle\cdot \mid \cdot\rangle_{H}$ denotes the scalar product in $H$ (which we suppose left linear, right antilinear in the complex case). When $p, q$ are conjugate $\left(\frac{1}{p}+\frac{1}{q}=1\right)$, we obtain a sesquilinear pairing

$$
\langle f, g\rangle=\int_{\Omega}\langle\langle f \mid g\rangle\rangle d \mu
$$

which gives rise to a canonical antilinear identification of $L_{q}(H)$ with $L_{p}(H)^{*}$ (if $1<p, q<\infty$; the case $p=1, q=\infty$ is more delicate); it is the usual duality pairing in the real spaces case. We have also

$$
\forall f \in L_{p}(H),\left\langle\left\langle f \mid u_{f}\right\rangle\right\rangle=N(f) .
$$

We say that two elements $f, g \in L_{p}(H)$ are orthogonal, and we write $f \perp g$ if $\langle\langle f|$ $g\rangle=0$. A related notation is the following. We set

$$
\{f \perp g\}=\{\omega \in \Omega \mid\langle\langle f \mid g\rangle\rangle(\omega)=0\}
$$

We have then $f \perp g \quad \Longleftrightarrow \quad\{f \perp g\} \doteq \Omega$.

Let $H, K$ two Hilbert spaces. We say that a linear operator $T: L_{p}(H) \rightarrow L_{p}(K)$ is $\Sigma$-modular iff $T(\varphi \cdot f)=\varphi$.Tf for every $f \in L_{p}(H)$ and $\varphi \in L_{\infty}(\Omega, \Sigma, \mu)$. It is modularly contractive, resp. modularly isometric iff $N(T f) \leq N(f)$, resp. $N(T f)=$ $N(f)$ for every $f \in L_{p}(H)$ : it is then automatically $\Sigma$-modular (and, of course, contractive, resp. isometric). If $H$ is separable, then a modularly contractive, resp. modularly isometric operator $T$ is associated with a measurable family of contractions, resp. isometries $T_{\omega}: H \rightarrow K$.

Let $\mathcal{F}$ be a sub-sigma-algebra of $\Sigma$; a linear subspace $Z$ of $L_{p}(H)$ is a $L_{\infty}(\mathcal{F})$ submodule iff $\varphi \cdot f \in Z$ for every $f \in Z$ and $\varphi \in L_{\infty}(\Omega, \mathcal{F}, \mu)$. To every $f \in L_{p}(H)$ we associate the bounded $\Sigma$-modular operator:

$$
E_{f}: L_{p}(H) \rightarrow L_{p}(H), \quad g \mapsto\left\langle\left\langle g \mid u_{f}\right\rangle\right\rangle u_{f} .
$$


We have $N\left(E_{f} g\right)=\left|\left\langle\left\langle g \mid u_{f}\right\rangle\right\rangle\right| \mathbf{1}_{\mathbf{V S}(f)} \leq N(g)$, hence $E_{f}$ is modularly contractive.

We have clearly $E_{f}(f)=N(f) u_{f}=f$. Consequently for every $\varphi \in L_{\infty}$, we have

$$
E_{f}\left((\varphi N(f)) \cdot u_{f}\right)=E_{f}(\varphi f)=\varphi f=(\varphi N(f)) \cdot u_{f}
$$

and by density we deduce that $E_{f}\left(\psi \cdot u_{f}\right)=\psi \cdot u_{f}$ for every $\psi \in L_{p}$. In particular $E_{f}\left(E_{f} g\right)=E_{f} g$, so $E_{f}$ is a projection (with range $R\left(E_{f}\right)=L_{p}(\Omega) \cdot u_{f}$ ). It is not hard to see that $R\left(E_{f}\right)$ is exactly the closed $L_{\infty}(\Sigma)$-submodule generated by $f$. Note also that if $f, g \in L_{p}(H)$,

$$
f \perp g \quad \Longleftrightarrow \quad E_{f} g=0 \quad \Longleftrightarrow \quad E_{g} f=0 .
$$

\subsection{Orthogonal projections}

We end this section by considering a special class of contractive projections, namely the orthogonal ones. A projection $Q$ in $L_{p}(H)$ is said to be orthogonal if $(f-Q f) \perp Q f$ for every $f \in L_{p}(H)$. Such a projection is trivially modularly contractive since

$$
N(f)^{2}=N(Q f)^{2}+N((I-Q) f)^{2} \geq N(Q f)^{2} .
$$

Note that by polarization we have for every $f, g \in L_{p}(H)$ :

$$
\langle\langle f \mid g\rangle\rangle=\langle\langle Q f \mid Q g\rangle\rangle+\langle\langle(I-Q) f \mid(I-Q) g\rangle\rangle
$$

Replacing $g$ by $Q g$, we have

$$
\langle\langle f \mid Q g\rangle\rangle=\langle\langle Q f \mid Q g\rangle\rangle
$$

that is $(I-Q) f \perp Q g$; hence $\operatorname{ker} Q=R(I-Q) \perp R(Q)$.

Conversely if $f \perp R(I-Q)$ then $f-Q f \perp R(I-Q)$ and in particular $f-Q f \perp$ $f-Q f$, i.e. $f=Q f \in R(Q)$. Hence $R(Q)=\operatorname{ker} Q^{\perp}:=\left\{f \in L_{p}(H) \mid f \perp \operatorname{ker} Q\right\}$ and similarly (exchanging the roles of $Q$ and $I-Q$ ) we have: $\operatorname{ker} Q=R(Q)^{\perp}$.

If $A$ is a subset of $L_{p}(H)$ then $A^{\perp}$ is a closed $L_{\infty}(\Sigma)$-submodule of $L_{p}(H)$. In particular the range of any orthogonal projection in $L_{p}(H)$ is a closed $L_{\infty}(\Sigma)$-submodule. The converse is true:

Lemma 1.1. If $Z$ is a closed $L_{\infty}(\Sigma)$-submodule of $L_{p}(\Omega, \Sigma, \mu ; H)$ there exists a unique orthogonal projection $Q_{Z}$ in $L_{p}(H)$ with range $Z$.

Proof. Let $\left(f_{\alpha}\right)_{\alpha \in A}$ be a maximal family of pairwise orthogonal non zero elements of $Z$. For every family $\left(\varphi_{\alpha}\right)_{\alpha}$ in $L_{p}(\Omega)$ and every finite subset $B$ of $A$ we have

$$
\left\|\sum_{\alpha \in B} \varphi_{\alpha} u_{f_{\alpha}}\right\|_{L_{p}(H)}=\left\|N\left(\sum_{\alpha \in B} \varphi_{\alpha} u_{f_{\alpha}}\right)\right\|_{p}=\left\|\left(\sum_{\alpha \in B} \mathbf{1}_{\mathbf{V S}\left(f_{\alpha}\right)}\left|\varphi_{\alpha}\right|^{2}\right)^{1 / 2}\right\|_{p} .
$$


Hence, by Cauchy's criterion, $\sum_{\alpha \in A} \varphi_{\alpha} u_{f_{\alpha}}$ converges in $L_{p}(H)$ iff $\left(\sum_{\alpha \in A} \mathbf{1}_{\mathbf{V S}\left(f_{\alpha}\right)}\right.$ $\left.\left|\varphi_{\alpha}\right|^{2}\right)^{1 / 2}$ exists in $L_{p}$ and

$$
\left\|\sum_{\alpha \in A} \varphi_{\alpha} u_{f_{\alpha}}\right\|_{L_{p}(H)}=\left\|\left(\sum_{\alpha \in A} \mathbf{1}_{\mathbf{V S}\left(f_{\alpha}\right)}\left|\varphi_{\alpha}\right|^{2}\right)^{1 / 2}\right\|_{p} .
$$

If now $f \in L_{p}(H)$ and $B$ is a finite subset of $A$ we have

$$
\begin{aligned}
N\left(\sum_{\alpha \in B}\left\langle\left\langle f \mid u_{f_{\alpha}}\right\rangle\right\rangle u_{f_{\alpha}}\right)^{2}= & \sum_{\alpha \in B}\left|\left\langle\left\langle f \mid u_{f_{\alpha}}\right\rangle\right\rangle\right|^{2} \\
& =\left\langle\left\langle f, \sum_{\alpha \in B}\left\langle\left\langle f \mid u_{f_{\alpha}}\right\rangle\right\rangle u_{f_{\alpha}}\right\rangle\right\rangle \leq N(f) N\left(\sum_{\alpha \in B}\left\langle\left\langle f \mid u_{f_{\alpha}}\right\rangle\right\rangle u_{f_{\alpha}}\right),
\end{aligned}
$$

whence

$$
N\left(\sum_{\alpha \in B}\left\langle\left\langle f \mid u_{f_{\alpha}}\right\rangle\right\rangle u_{f_{\alpha}}\right)=\left(\sum_{\alpha \in B}\left|\left\langle\left\langle f \mid u_{f_{\alpha}}\right\rangle\right\rangle\right|^{2}\right)^{1 / 2} \leq N(f),
$$

so

$$
\left(\sum_{\alpha \in A}\left|\left\langle\left\langle f \mid u_{f_{\alpha}}\right\rangle\right\rangle\right|^{2}\right)^{1 / 2} \leq N(f) .
$$

Consequently $Q f:=\sum_{\alpha \in A}\left\langle\left\langle f \mid u_{f_{\alpha}}\right\rangle u_{f_{\alpha}}=\sum_{\alpha \in A} E_{f_{\alpha}} f\right.$ converges in $L_{p}(H)$ (with $\|Q f\| \leq\|f\|)$. Since $R\left(E_{f_{\alpha}}\right)$ is the closed $L_{\infty}(\Sigma)$-submodule generated by $f_{\alpha}$, we have $R\left(E_{f_{\alpha}}\right) \subset Z$ for each $\alpha$ and consequently $Q f \in Z$ for every $f \in L_{p}(H)$. The map $Q$ is modular for the action of $L_{\infty}(\Omega)$, and clearly $Q f_{\beta}=f_{\beta}$ for every $\beta \in A$. It results easily that $Q f=f$ for every $f=\sum_{\alpha \in A} \varphi_{\alpha} u_{f_{\alpha}}$ (when this series converges), i.e. $Q$ is a contractive projection in $L_{p}(H)$ with range

$$
\begin{aligned}
R(Q) & =\left\{\sum_{\alpha \in A} \varphi_{\alpha} u_{f_{\alpha}} \mid\left(\sum_{\alpha}\left|\varphi_{\alpha}\right|^{2}\right)^{1 / 2} \in L_{p}(\Omega)\right\} \\
& =\left\{\sum_{\alpha \in A} \psi_{\alpha} f_{\alpha} \mid\left(\sum_{\alpha}\left|\psi_{\alpha}\right|^{2} N\left(f_{\alpha}\right)^{2}\right)^{1 / 2} \in L_{p}(\Omega)\right\} .
\end{aligned}
$$

Since clearly $\left\langle\left\langle Q f \mid f_{\alpha}\right\rangle\right\rangle=\left\langle\left\langle f \mid f_{\alpha}\right\rangle\right\rangle$ for every $\alpha \in A$ we have $(f-Q f) \perp f_{\alpha}$ for every $\alpha \in A$. By maximality of the system $\left(f_{\alpha}\right)$ we deduce that

$$
f=Q f \text { for every } f \in Z
$$

so $R(Q)$ contains $Z$, hence coincides with $Z$. Note also that $f-Q f \perp Z$ for all $f \in L_{p}(H)$, and so $Q$ is orthogonal.

The unicity of the orthogonal projection onto $Z$ is a consequence of the fact that its image and kernel are uniquely determined $\left(R(Q)=Z\right.$ and $\left.\operatorname{ker} Q=Z^{\perp}\right)$. 


\section{Preliminary results: the case $p=1$}

Lemma 2.1. Let $P$ be a contractive projection in $L_{1}(H)$. Then for every $f \in R(P)$ we have

$$
P E_{f}=E_{f} P E_{f}
$$

Proof. For every $\varphi \in L_{1}(\Omega)$ with $0 \leq \varphi \leq N(f)$ we have

$$
\begin{aligned}
\|f\|-\left\|\varphi \cdot u_{f}\right\| & =\int N(f) d \mu-\int N\left(\varphi u_{f}\right) d \mu=\int(N(f)-\varphi) d \mu \\
& =\left\|(N(f)-\varphi) \cdot u_{f}\right\|=\left\|f-\varphi \cdot u_{f}\right\| \\
& \geq\left\|P\left(f-\varphi \cdot u_{f}\right)\right\|=\left\|f-P\left(\varphi \cdot u_{f}\right)\right\| \\
& \geq\|f\|-\left\|P\left(\varphi \cdot u_{f}\right)\right\| \\
& \geq\|f\|-\left\|\varphi \cdot u_{f}\right\| .
\end{aligned}
$$

Hence all the inequalities are equalities, and in particular

$$
\left\|f-P\left(\varphi \cdot u_{f}\right)\right\|=\|f\|-\left\|P\left(\varphi \cdot u_{f}\right)\right\|,
$$

that is,

$$
\int N\left(f-P\left(\varphi \cdot u_{f}\right)\right) d \mu=\int\left[N(f)-N\left(P\left(\varphi \cdot u_{f}\right)\right)\right] d \mu .
$$

Note that the function in the left-hand integral is greater than the one in the righthand integral. Thus,

$$
N\left(f-P\left(\varphi \cdot u_{f}\right)\right)=N(f)-N\left(P\left(\varphi \cdot u_{f}\right)\right)
$$

(equality as elements of $L_{1}(\Omega)$ ). Since $H$ is strictly convex this implies that

$$
P\left(\varphi \cdot u_{f}\right)=\alpha \cdot f
$$

for some $\alpha \in L_{\infty}^{+}(\Omega)$. Hence

$$
E_{f} P\left(\varphi \cdot u_{f}\right)=E_{f}(\alpha \cdot f)=\alpha \cdot f=P\left(\varphi \cdot u_{f}\right)
$$

This property has been proved for $\varphi \in L_{1}(\Omega)$ with $0 \leq \varphi \leq N(f)$; it is extended by linearity and density to every $\varphi \in L_{1}(\Omega)$. In particular if we take $\varphi=\left\langle\left\langle h \mid u_{f}\right\rangle\right\rangle$, we obtain

$$
\forall h \in L_{1}(H), \quad E_{f} P E_{f} h=P E_{f} h,
$$

that is, $E_{f} P E_{f}=P E_{f}$.

Lemma 2.2. Let $P$ be a contractive projection in $L_{1}(H)$. Then for every $f, g \in R(P)$ we have: $E_{g} f \in R(P)$. In other words $E_{g} P=P E_{g} P$. 
Proof. We have $\left(f-E_{g} f\right) \perp g$, while (by Lemma 2.1) $E_{g} f-P E_{g} f=E_{g}\left(f-P E_{g} f\right) \in$ $L_{1}(\Omega) \cdot u_{g}$. Hence $\left(f-E_{g} f\right) \perp\left(E_{g} f-P E_{g} f\right)$. It results that

$$
N\left(f-P E_{g} f\right)=\left[N\left(f-E_{g} f\right)^{2}+N\left(\left(E_{g} f-P E_{g} f\right)^{2}\right]^{1 / 2} \geq N\left(f-E_{g} f\right) .\right.
$$

Hence:

$$
\begin{aligned}
\left\|f-P E_{g} f\right\| & \geq\left\|f-E_{g} f\right\| \\
& \geq\left\|P\left(f-E_{g} f\right)\right\| \\
& =\left\|f-P E_{g} f\right\|
\end{aligned}
$$

Hence the inequalities are equalities. In view of (4), the equality $\left\|f-P E_{g} f\right\|=$ $\left\|f-E_{g} f\right\|$ implies

$$
N\left(f-P E_{g} f\right)=\left[N\left(f-E_{g} f\right)^{2}+N\left(\left(E_{g} f-P E_{g} f\right)^{2}\right]^{1 / 2}=N\left(f-E_{g} f\right),\right.
$$

which implies in turn that $N\left(E_{g} f-P E_{g} f\right)=0$, that is $E_{g} f=P E_{g} f$. So $E_{g} f \in$ $R(P)$.

\section{Preliminary results: the case $p>1$}

Notations. Let $p_{*}$ be the conjugate exponent of $p$. If $T: L_{p}(H) \rightarrow L_{p}(H)$ is a bounded operator, we define its adjoint $T^{*}: L_{p_{*}}(H) \rightarrow L_{p_{*}}(H)$ by

$$
\forall f \in L_{p_{*}}(H), \forall g \in L_{p}(H) \quad\left\langle T^{*} f, g\right\rangle=\langle f, T g\rangle,
$$

where $\langle\cdot, \cdot\rangle$ denotes the sesquilinear pairing given by eq. (3).

If $f \in L_{p}(H), f \neq 0$, let $J f \in L_{p_{*}}(H)$ be the unique norm-one element such that $\langle f, J f\rangle=\|f\|$. In fact it will be easier to consider the $(p-1)$-homogeneous functional $J_{p}(h)=\|h\|^{p-1} J(h)$. We have $J_{p}(h)=N(h)^{p-1} \cdot u_{h}=N(h)^{p-2} h$, hence $J_{p}$ is random direction preserving. Note that $p J_{p}$ is the derivative of the $p^{\text {th }}$ power of the norm.

Lemma 3.1. Let $1<p<\infty, p \neq 2$, and $P$ be a contractive projection in $L_{p}(H)$. Then for every $f, g \in R(P)$ the function $F(f, g):=\operatorname{sgn}\langle\langle g \mid f\rangle\rangle f+\gamma_{p} \mathbf{1}_{\{f \perp g\}} N(f) u_{g}$ belongs to $R(P)$, where $\gamma_{p}$ is a positive constant depending only on $p$.

Proof. a) Case $2<p<\infty$.

Recall that since $L_{p}(H)$ is smooth the duality map $J$ maps $R(P)$ into $R\left(P^{*}\right)$ (see e.g. [6, Lemma 4.8]); hence $J_{p}(f+t g) \in R\left(P^{*}\right)$ for every $t \geq 0$. The derivative $\frac{\partial}{\partial t} J_{p}(f+t g)$ exists at $t=0$ (since the norm to the power $p$ is twice differentiable) and it belongs to $R\left(P^{*}\right)$ too. We have

$$
\frac{\partial}{\partial t} J_{p}(f+t g)=N(f+t g)^{p-2} g+\left(\frac{p-2}{2} \frac{\partial}{\partial t} N(f+t g)^{2}\right) N(f+t g)^{p-4}(f+t g) .
$$


Hence

$$
\begin{aligned}
A(f, g):=\left.\frac{\partial}{\partial t} J_{p}(f+t g)\right|_{t=0} & =N(f)^{p-2} g+(p-2) \operatorname{Re}(\langle\langle f \mid g\rangle\rangle) N(f)^{p-4} f \\
& =N(f)^{p-2}\left[g+(p-2) \operatorname{Re}\left(\left\langle\left\langle u_{f}, g\right\rangle\right\rangle\right) u_{f}\right] \in R\left(P^{*}\right)
\end{aligned}
$$

In the complex case, replacing $f$ by if, we obtain

$$
B(f, g):=N(f)^{p-2}\left[g-i(p-2) \operatorname{Im}\left(\left\langle\left\langle u_{f}, g\right\rangle\right\rangle\right) u_{f}\right] \in R\left(P^{*}\right)
$$

adding

$$
N(f)^{p-2}\left[2 g+(p-2)\left\langle\left\langle g, u_{f}\right\rangle\right\rangle u_{f}\right] \in R\left(P^{*}\right)
$$

With $E_{f} g=\left\langle\left\langle g, u_{f}\right\rangle\right\rangle u_{f}$ we obtain

$$
N(f)^{p-2}\left[2\left(g-E_{f} g\right)+p E_{f} g\right] \in R\left(P^{*}\right) .
$$

In the case of a real space (5) is valid without the symbol Re and we obtain

$$
N(f)^{p-2}\left[\left(g-E_{f} g\right)+(p-1) E_{f} g\right] \in R\left(P^{*}\right) .
$$

If $h \in R\left(P^{*}\right)$ then $J_{p_{*}} h=N(h)^{p_{*}-1} u_{h} \in R(P)$, hence if we set $T g=\alpha_{p}\left(g-E_{f} g\right)+$ $E_{f} g$, with $\alpha_{p}=\frac{2}{p}$ in the complex case, $\alpha_{p}=\frac{1}{p-1}$ in the real case, we obtain:

$$
\Phi(g):=N(f)^{(p-2)\left(p_{*}-1\right)} N(T g)^{\left(p_{*}-1\right)} u_{T g} \in R(P) .
$$

Since $T$ is $\Sigma$-modular we have $u_{T\left(\varphi \cdot u_{h}\right)}=\mathbf{1}_{\operatorname{Supp} \varphi} \cdot u_{T h}$ for every $h \in L_{p}(H)$ and $\varphi \in L_{p}$, and more generally $u_{T^{k}\left(\varphi \cdot u_{h}\right)}=\mathbf{1}_{\operatorname{Supp} \varphi} \cdot u_{T^{k} h}$ for every $k \geq 1$. It is easily deduced that: $u_{T^{k} \Phi(g)}=\mathbf{1}_{\mathbf{V S}(f)} \cdot u_{T^{k+1} g}$ for every $k \geq 0$. Then

$$
\begin{aligned}
u_{\Phi^{n}(g)} & =u_{\Phi\left(\Phi^{n-1}(g)\right)}=\mathbf{1}_{\mathbf{V S}(f)} \cdot u_{T \Phi^{n-1}(g)} \\
& =\mathbf{1}_{\mathbf{V S}(f)} \cdot u_{T \Phi\left(\Phi^{n-2}(g)\right)}=\mathbf{1}_{\mathbf{V S}(f)} \cdot u_{T^{2} \Phi^{n-2}(g)} \cdots \\
& =\mathbf{1}_{\mathbf{V S}(f)} \cdot u_{T^{n} g}
\end{aligned}
$$

for every $n \geq 1$. If $E_{f} g(\omega) \neq 0$ we have

$$
u_{T^{n} g}(\omega)=\frac{\alpha_{p}^{n}\left(g-E_{f} g\right)(\omega)+E_{f} g(\omega)}{N\left(\alpha_{p}^{n}\left(g-E_{f} g\right)+E_{f} g\right)(\omega)} \longrightarrow \frac{E_{f} g(\omega)}{N\left(E_{f} g\right)(\omega)}=u_{E_{f} g}(\omega)
$$

(norm convergence in $H$ ) while if $E_{f} g(\omega)=0$

$$
u_{T^{n} g}(\omega)=\frac{\left(g-E_{f} g\right)(\omega)}{N\left(\left(g-E_{f} g\right)\right)(\omega)}=u_{\left(g-E_{f} g\right)}(\omega)=u_{g}(\omega) .
$$

Since $g-E_{f} g \perp E_{f} g$ we have $N(T g) \leq N(g)$. Hence

$$
N(\Phi(g))=N(f)^{2-p_{*}} N(T g)^{p_{*}-1} \leq N(f)^{2-p_{*}} N(g)^{p_{*}-1} .
$$


In particular

$$
N(\Phi(g)) \leq \max (N(f), N(g)) .
$$

Reiterating (8) we obtain for every $n \geq 1$

$$
N\left(\Phi^{n}(g)\right) \leq N(f)^{\left(2-p_{*}\right) \Sigma_{k=0}^{n-1}\left(p_{*}-1\right)^{k}} N(g)^{\left(p_{*}-1\right)^{n}}=N(f)^{1-\left(p_{*}-1\right)^{n}} N(g)^{\left(p_{*}-1\right)^{n}} .
$$

Since $0<p_{*}-1<1$ we obtain

$$
\varlimsup_{n \rightarrow \infty} N\left(\Phi^{n}(g)\right) \leq \mathbf{1}_{\mathbf{V S}(g)} N(f) .
$$

We try now to be more precise. If $E_{f} g(\omega)=0$ we have $N(T g)(\omega)=\alpha_{p} N(g)(\omega)$. Hence

$$
N(\Phi(g))(\omega)=N(f)(\omega)^{2-p_{*}}\left(\alpha_{p} N(g)(\omega)\right)^{p_{*}-1} .
$$

Moreover, since in this case $u_{\Phi^{n}(g)}(\omega)=u_{g}(\omega)$, we have $E_{f} \Phi^{n}(g)(\omega)=0$ for every $n$, and we can reiterate. We obtain

$$
N\left(\Phi^{n}(g)\right)(\omega)=\left(\alpha_{p}^{p_{*}-1} N(f)(\omega)^{\left(2-p_{*}\right)}\right)^{\sum_{k=0}^{n-1}\left(p_{*}-1\right)^{k}} N(g)(\omega)^{\left(p_{*}-1\right)^{n}} .
$$

Hence

$$
\begin{aligned}
\lim _{n \rightarrow \infty} N\left(\Phi^{n}(g)\right)(\omega) & =\alpha_{p}^{\left(p_{*}-1\right) /\left(2-p_{*}\right)} \mathbf{1}_{\mathbf{V S}(g)}(\omega) N(f)(\omega) \\
& =\alpha_{p}^{1 /(p-2)} \mathbf{1}_{\mathbf{V S}(g)}(\omega) N(f)(\omega) .
\end{aligned}
$$

If now $E_{f}(g)(\omega) \neq 0$, we have also $E_{f}\left(\Phi^{n}(g)\right)(\omega) \neq 0$ for every $n \geq 0$. Set

$$
\beta_{n}(\omega)=\frac{N\left(E_{f} \Phi^{n}(g)\right)(\omega)}{N\left(\Phi^{n}(g)\right)(\omega)}
$$

We have then

$$
N\left(T \Phi^{n}(g)\right)(\omega) \geq \beta_{n}(\omega) N\left(\Phi^{n}(g)\right)(\omega)
$$

and consequently:

$$
N\left(\Phi^{n+1}(g)\right)(\omega) \geq N(f)^{2-p_{*}}\left(\beta_{n}(\omega) N\left(\Phi^{n}(g)(\omega)\right)^{p_{*}-1} .\right.
$$

On the other hand

$$
\beta_{n}(\omega)=\left|\left\langle\left\langle u_{\Phi^{n}(g)}, u_{f}\right\rangle\right\rangle(\omega)\right|=\left|\left\langle\left\langle u_{T^{n}(g)}, u_{f}\right\rangle\right\rangle(\omega)\right|=\frac{N\left(E_{f} T^{n}(g)\right)(\omega)}{N\left(T^{n}(g)\right)(\omega)}=\frac{N\left(E_{f} g\right)(\omega)}{N\left(T^{n}(g)\right)(\omega)}
$$

and since $N\left(T^{n} g\right)=\left(\alpha_{p}^{2 n} N\left(g-E_{f} g\right)^{2}+N\left(E_{f} g\right)^{2}\right)^{1 / 2} \searrow N\left(E_{f} g\right)$ pointwise $\left(\right.$ as $\left.\alpha_{p}<1\right)$ we have $\beta_{n}(\omega) \nearrow 1$ on the set $\left\{\omega \mid E_{f} g(\omega) \neq 0\right\}$. Reiterating (12) from the step $n=n_{0}$ we obtain then

$$
\varliminf_{n \rightarrow \infty} N\left(\Phi^{n}(g)\right)(\omega) \geq\left(\beta_{n_{0}}(\omega)\right)^{1 /(p-2)} \mathbf{1}_{\mathbf{V S}\left(\Phi_{n_{0}}(g)\right)}(\omega) N(f)(\omega)
$$


and letting $n_{0} \rightarrow \infty$, we have, since $\mathbf{V S}\left(\Phi_{n}(g)\right)=\mathbf{V S}(g) \cap \mathbf{V S}(f)$ for every $n$,

$$
\varliminf_{n \rightarrow \infty} N\left(\Phi^{n}(g)\right)(\omega) \geq \mathbf{1}_{\mathbf{V S}(g)}(\omega) N(f)(\omega) .
$$

From (6), (7), (7’), and (11), (10), (13) we deduce that

$$
\Phi^{n}(g) \rightarrow N(f)\left[u_{E_{f}(g)}+\alpha_{p}^{1 /(p-2)} \mathbf{1}_{\{f \perp g\}} u_{g}\right]
$$

almost everywhere in $H$-norm, hence in $L_{p}(H)$-norm by $(9)$ and Lebesgue's Theorem. Hence the right-hand member of (14) belongs to $R(P)$. Since $u_{E_{f} g}=\operatorname{sgn}\langle\langle g \mid f\rangle\rangle u_{f}$ the right member of (14) can be written

$$
\operatorname{sgn}\langle\langle g \mid f\rangle\rangle f+\gamma_{p} \mathbf{1}_{\{f \perp g\}} N(f) u_{g}=F_{p}(f, g)
$$

where we have set $\gamma_{p}=\alpha_{p}^{1 /(p-2)}$.

b) Case $1<p<2$.

This case is treated by duality. Set $\gamma_{p}=\gamma_{p_{*}}^{p_{*}-1}$ and define $F_{p}(f, g)$ by the formula (15). If $g=J_{p_{*}} g^{\prime}, f=J_{p_{*}} h^{\prime}$ with $f^{\prime}, g^{\prime} \in L_{p_{*}}(H)$ we have

$$
\operatorname{sgn}\langle\langle g \mid f\rangle\rangle=\operatorname{sgn}\left\langle\left\langle g^{\prime} \mid f^{\prime}\right\rangle\right\rangle \text {. }
$$

Hence

$$
\operatorname{sgn}\langle\langle g \mid f\rangle\rangle f=J_{p_{*}}\left(\operatorname{sgn}\left\langle\left\langle g^{\prime} \mid f^{\prime}\right\rangle\right\rangle f^{\prime}\right)
$$

and similarly

$$
N(f)=N\left(J_{p^{*}} f^{\prime}\right)=N\left(f^{\prime}\right)^{p_{*}-1} .
$$

Hence

$$
N(f) u_{g}=N\left(f^{\prime}\right)^{p_{*}-1} u_{g}=J_{p_{*}}\left(N\left(f^{\prime}\right) u_{g^{\prime}}\right) .
$$

Finally, since $\{f \perp g\}=\left\{f^{\prime} \perp g^{\prime}\right\}$ and $J_{p_{*}}$ is additive on elements with disjoint functional supports, and positively homogeneous of degree $p_{*}-1$,

$$
F_{p}(f, g)=J_{p_{*}}\left(F_{p_{*}}\left(f^{\prime}, g^{\prime}\right)\right)
$$

Then since $f^{\prime}=J_{p} f, g^{\prime}=J_{p} g$ belong to $R\left(P^{*}\right)$, the function $\left.F_{p_{*}}\left(f^{\prime}, g^{\prime}\right)\right)$ belongs to $R\left(P^{*}\right)$ too by the case $(\mathrm{a})$, and $F_{p}(f, g)$ belongs to $R(P)$.

Corollary 3.2. Let $p$ and $P$ be as in Lemma 3.1. Then for every $f, g \in R(P)$ the three elements $\operatorname{sgn}\langle\langle g \mid f\rangle\rangle f, \mathbf{1}_{\{f \perp g\}} f$ and $\mathbf{1}_{\{f \perp g\}} N(f) u_{g}$ belong to $R(P)$.

Proof. The set $\Lambda$ of scalars $\lambda$ such that the set $\left\{\omega \in \mathbf{V S}(f) \mid \frac{\langle\langle g \mid f\rangle\rangle(\omega)}{\langle\langle f \mid f\rangle\rangle(\omega)}=-\lambda\right\}$ has positive measure is at most countable. This set is also the set of $\lambda$ 's such that $\{(g+\lambda f) \perp f\} \cap \mathbf{V S}(f)$ has positive measure. Choose a sequence $\left(\varepsilon_{n}\right)$ of positive numbers not in $\Lambda \cup(-\Lambda)$ which converges to 0 . Then by Lemma 3.1

$$
\operatorname{sgn}\left\langle\left\langle g \pm \varepsilon_{n} f \mid f\right\rangle\right\rangle f \in R(P)
$$


for every $n \geq 1$. Since

$$
\operatorname{sgn}\left\langle\left\langle g \pm \varepsilon_{n} f \mid f\right\rangle\right\rangle(\omega) \rightarrow\left\{\begin{array}{ll}
\operatorname{sgn}\langle\langle g \mid f\rangle\rangle(\omega) & \text { if }\langle\langle g \mid f\rangle\rangle(\omega) \neq 0, \\
\pm 1 & \text { if }\langle\langle g \mid f\rangle\rangle(\omega)=0
\end{array} \text { and } f(\omega) \neq 0,\right.
$$

we have

$$
\operatorname{sgn}\langle\langle g \mid f\rangle\rangle f \pm \mathbf{1}_{\{f \perp g\}} f=\lim _{n} \operatorname{sgn}\left\langle\left\langle g \pm \varepsilon_{n} f \mid f\right\rangle\right\rangle f \in R(P)
$$

and consequently sgn $\langle\langle g \mid f\rangle\rangle f$ and $\mathbf{1}_{\{f \perp g\}} f$ belong to $R(P)$. Then $F_{p}(f, g)-\operatorname{sgn}\langle\langle g|$ $f\rangle f=\gamma_{p} \mathbf{1}_{\{f \perp g\}} N(f) u_{g}$ belongs to $R(P)$ too.

Corollary 3.3. Let $p$ and $P$ be as in Lemma 3.1. Then for every $f, g \in R(P)$ we have $\mathbf{1}_{\mathbf{V S}(g)} f \in R(P)$.

Proof. By Cor. 3.2, $h:=G(f, g):=\mathbf{1}_{\{f \perp g\}} N(f) u_{g}$ belongs to $R(P)$. Then $G(h, f)=$ $\mathbf{1}_{\{f \perp g\}} \mathbf{1}_{\left\{u_{g} \neq 0\right\}} N(f) u_{f}=\mathbf{1}_{\mathbf{V S}(g) \cap\{f \perp g\}} f$ belongs to $R(P)$ too. By Cor. 3.2, $f-$ $\mathbf{1}_{\{f \perp g\}} f=\mathbf{1}_{\{f \not g g\}} f \in R(P)$, thus $\mathbf{1}_{\mathbf{V S}(g)} f=\mathbf{1}_{\{f \not g g\}} f+\mathbf{1}_{\mathbf{V S}(g) \cap\{f \perp g\}} f \in R(P)$.

Remark 3.4. In the complex case, for every $f, g \in R(P)$ the elements $\operatorname{sgn}(\operatorname{Re}\langle\langle g \mid f\rangle\rangle) f$ and $\mathbf{1}_{\{\operatorname{sgn}(\operatorname{Re}\langle|g| f\rangle\rangle)=0\}} f$ belong to $R(P)$ too. Indeed, $L_{p}(H)$ is a real Hilbert-valued $L_{p}(K)$ space, where $K$ is the real vector space $H$ equipped with the scalar product $(x, y)_{K}=\operatorname{Re}(x \mid y)_{H}$. As a consequence, the element $\mathbf{1}_{\{\operatorname{Re} e\langle g \mid f\rangle\rangle>0\}} f=\frac{1}{2}(\operatorname{sgn} \operatorname{Re}\langle\langle g|$ $\left.f\rangle\rangle+\mathbf{1}_{\{\operatorname{sgn}(\operatorname{Re}\langle\langle g \mid f\rangle\rangle) \neq 0\}}\right) f$ belongs to $R(P)$.

Lemma 3.5. Let $p$ and $P$ be as in Lemma 3.1. For every $f, g \in R(P)$ denote by $\Sigma_{f, g}$ the $\sigma$-field generated by the element $\frac{\langle g|f|\rangle}{\langle\langle f \mid f\rangle\rangle}$. Then for every $\Sigma_{f, g}$-measurable function $\varphi$ such that $\varphi \cdot N(f) \in L_{p}(\Omega, \Sigma, \mu)$, the element $\varphi \cdot f$ belongs to $R(P)$.

Proof. Since $R(P)$ is a closed linear subspace, it is sufficient to prove this for indicator functions of $\Sigma_{f, g}$-measurable sets. The sigma-algebra $\Sigma_{f, g}$ is generated by the sets $\left\{\frac{\operatorname{Re}\langle\langle g \mid f\rangle\rangle}{\langle\langle f \mid f\rangle\rangle}>\lambda\right\},\left\{-\frac{\operatorname{Re}\langle g \mid f\rangle\rangle}{\langle\langle f \mid f\rangle\rangle}>\lambda\right\},\left\{\frac{\operatorname{Im}\langle\langle g \mid f\rangle\rangle}{\langle\langle f \mid f\rangle}>\lambda\right\}$, and $\left\{-\frac{\operatorname{Im}\langle\langle g \mid f\rangle\rangle}{\langle\langle f \mid f\rangle\rangle}>\lambda\right\}, \lambda \in \mathbb{R}_{+}$. If $A_{f, g, \lambda}=\left\{\frac{\operatorname{Re}\langle|g| f\rangle\rangle}{\langle\langle f \mid f\rangle\rangle}>\lambda\right\}$ we have $A_{f, g, \lambda}=\{\operatorname{Re}\langle\langle g-\lambda f \mid f\rangle\rangle>0\}$, hence $\mathbf{1}_{A_{f, g, \lambda}} f \in$ $R(P)$ by Rem. 3.4. The conclusion is the same for the three others kinds of sets (replacing $g$ by $-g$ or $\pm i g$ ). Now if $\mathbf{1}_{B} f \in R(P)$ then $A_{f, g, \lambda} \cap B=A_{f^{\prime}, g, \lambda}$ with $f^{\prime}=\mathbf{1}_{B} f$, hence $\mathbf{1}_{A_{f, g, \lambda} \cap B} f=\mathbf{1}_{A_{f^{\prime}, g, \lambda}} f^{\prime} \in R(P)$. It results that the class $\mathcal{C}$ of the sets $A \in \Sigma$ such that $\mathbf{1}_{A} f \in R(P)$ contains finite intersections of sets of the four preceding types. Since $\mathcal{C}$ is closed by complementation and monotone limits, it contains the sigma-algebra $\Sigma_{f, g}$.

Corollary 3.6. Let $p$ and $P$ be as in Lemma 3.1. For every $f \in R(P)$ we have $E_{f} P=P E_{f}$. 
Proof. Let $g \in R(P)$. Applying Lemma 3.5 to the function $\varphi=\frac{\langle\langle g \mid f\rangle\rangle}{\langle\langle f \mid f\rangle\rangle}$ we obtain that $E_{f} g \in R(P)$. Hence for every $h \in L_{p}(H)$, we have $E_{f} P h \in R(P)$, i.e. $E_{f} P h=$ $P E_{f} P h$; thus $E_{f} P=P E_{f} P$. Similarly, reasoning with the contractive projection $P^{*}$ in $L_{p_{*}}(H)$, and the element $J_{p} f$ of $R\left(P^{*}\right)$, we have $E_{J_{p} f} P^{*}=P^{*} E_{J_{p} f} P^{*}$. Dualizing we obtain $P E_{J_{p} f}^{*}=P E_{J_{p} f}^{*} P$. We claim that $E_{f}^{*}=E_{J_{p} f}$. This will show that $P E_{f}=P E_{f} P=E_{f} P$. Let us show this claim. Since $u_{J_{p} f}=u_{f}$, we have for every $g \in L_{p}(H)$ and $h^{\prime} \in L_{p_{*}}(H)$

$$
\begin{aligned}
\left\langle E_{f} g, h^{\prime}\right\rangle & =\int\left\langle\left\langle E_{f} g, h^{\prime}\right\rangle\right\rangle d \mu=\int\left\langle\left\langle\left\langle\left\langle g, u_{f}\right\rangle\right\rangle u_{f}, h^{\prime}\right\rangle\right\rangle d \mu \\
& =\int\left\langle\left\langle g, u_{f}\right\rangle\right\rangle\left\langle\left\langle u_{f}, h^{\prime}\right\rangle\right\rangle d \mu \\
& =\int\left\langle\left\langle g,\left\langle\left\langle h^{\prime}, u_{f}\right\rangle\right\rangle u_{f}\right\rangle\right\rangle d \mu \\
& =\int\left\langle\left\langle g, E_{J_{p} f} h^{\prime}\right\rangle\right\rangle d \mu=\left\langle g, E_{J_{p} f} h^{\prime}\right\rangle
\end{aligned}
$$

Remark. The preceding proof of Cor. 3.6 is essentially a real one. In the complex case it can be replaced by a shorter one, of more algebraic nature, due to Arazy and Friedman in the context of spaces $C_{p}$ (see [2]). It seemed interesting to us to reproduce this proof in the Annex (see $\S 6$ ), after simplifying it considerably by eliminating the unnecessary non-commutative apparatus.

\section{The range of a contractive projection}

This section is devoted to the proof of Thm. 0.1, which consists in four lemmas.

Lemma 4.1. The closed $L_{\infty}(\Sigma)$-module $Z$ generated by $R(P)$ in $L_{p}(H)$ is generated (as $L_{\infty}$-module) by a family $\left(f_{\alpha}\right)_{\alpha \in A}$ of pairwise orthogonal elements of $R(P)$. We have in fact a Schauder (orthogonal) decomposition

$$
Z=\bigoplus_{\alpha \in A} L_{p}(\Omega) \cdot u_{f_{\alpha}}
$$

Proof. Let $\left(f_{\alpha}\right)_{\alpha \in A}$ be a maximal family of pairwise orthogonal non zero elements of $R(P)$ and $Z_{0}$ be the closed $L_{\infty}(\Sigma)$-submodule generated by the family $\left(f_{\alpha}\right)_{\alpha \in A}$. Let $Q_{Z_{0}}$ be the orthogonal projection onto $Z_{0}$. By the proof of Lemma 1.1 we know that $Q_{Z_{0}}=\sum_{\alpha \in A} E_{f_{\alpha}}$ (convergence in strong operator topology). Hence, by Lemma 2.2 if $p=1$, resp. Cor. 3.6 if $p>1, Q_{Z_{0}} f \in R(P)$ for every $f \in R(P)$. Since $Q_{Z_{0}}$ is orthogonal and $f_{\alpha} \in R\left(Q_{Z_{0}}\right)$ we have $\left(f-Q_{Z_{0}} f\right) \perp f_{\alpha}$ for every $\alpha \in A$. By maximality of the system $\left(f_{\alpha}\right)$ we deduce that

$$
f=Q_{Z_{0}} f \text { for every } f \in R(P)
$$


i.e. $Q_{Z_{0}} P=P$. Then $Z_{0}=R\left(Q_{Z_{0}}\right)$ is a closed $L_{\infty}$-module containing $R(P)$ and generated by a subset of $R(P)$; hence it coincides with the closed $L_{\infty}$-module generated by $R(P)$.

Lemma 4.2. There exists a sub- $\sigma$-algebra $\mathcal{F}$ of $\Sigma$ containing the vectorial function supports of all elements of $R(P)$ such that for every $f \in R(P)$ and $\varphi \in L_{p}(\Omega, \Sigma, \mu)$, the product $\varphi \cdot u_{f}$ belongs to $R(P)$ iff $\mathbf{1}_{\mathbf{V S}(f)} N(f)^{-1} \varphi$ is $\mathcal{F}$-measurable. In particular $R(P)$ is a $L_{\infty}(\Omega, \mathcal{F}, \mu)$-submodule.

Proof. Since $P E_{f}=E_{f} P E_{f}$ by Lemma 2.1 (if $p=1$ ) or by Cor. 3.6 (if $p>1$ ), we have $P\left(\varphi \cdot u_{f}\right) \in L_{p}(\Omega) \cdot u_{f}$ for every $f \in R(P)$ and $\varphi \in L_{p}(\Omega, \Sigma, \mu)$. We may write $P\left(\varphi \cdot u_{f}\right)=\left(\widetilde{P}_{f} \varphi\right) \cdot u_{f}$, with $\operatorname{Supp}\left(\widetilde{P}_{f} \varphi\right) \subset \mathbf{V S}(f)$. Clearly $\widetilde{P}_{f}$ is linear, $\widetilde{P}_{f}^{2}=\widetilde{P}_{f}$ and

$$
\left\|\widetilde{P}_{f} \varphi\right\|_{p}=\left\|P\left(\varphi \cdot u_{f}\right)\right\| \leq\left\|\varphi \cdot u_{f}\right\| \leq\|\varphi\|_{p}
$$

hence $\widetilde{P}_{f}$ is a contractive projection in $L_{p}(\Omega, \Sigma, \mu)$. Moreover $\widetilde{P}_{f}(N(f))=N(f)$ and $\widetilde{P}_{f} \psi=0$ for every $\psi \in L_{p}(\Omega, \Sigma, \mu)$ disjoint from $N(f)$.

It results from Douglas' theorem (in case $p=1$ ) or Andô's theorem (in case $p>1$ ) that $\widetilde{P}_{f}$ is positive and

$$
\widetilde{P}_{f}(\varphi)=N(f) \mathbb{E}_{\nu_{f}}^{\mathcal{F}_{f}}\left(\frac{\mathbf{1}_{\operatorname{Supp}(N(f))} \varphi}{N(f)}\right)
$$

where $\mathbb{E}_{\nu_{f}}^{\mathcal{F}_{f}}$ is the conditional expectation with respect to some subalgebra $\mathcal{F}_{f}$ of $\Sigma$ containing $\mathbf{V S}(f)$ and to the measure $\nu_{f}=N(f)^{p} d \mu$. (We may assume that $\Omega \backslash \mathbf{V S}(f)$ is an atom of $\left.\mathcal{F}_{f}\right)$. In particular $L_{p}(\Omega, \Sigma, \mu) \cdot u_{f} \cap R(P)=L_{p}\left(\Omega, \mathcal{F}_{f}, \nu_{f}\right) \cdot f$ is a $L_{\infty}\left(\Omega, \mathcal{F}_{f}, \mu\right)$-module.

Let us denote $\mathbb{E}^{f} \psi=\mathbb{E}_{\nu_{f}}^{\mathcal{F}_{f}}\left(\mathbf{1}_{\mathbf{V S}(f)} \psi\right)$, we have then $P(\psi \cdot f)=\mathbb{E}^{f}(\psi) \cdot f$ for every $\psi \in L_{\infty}(\Omega, \Sigma, \mu)$. Let now $f, g \in R(P)$. If $g=h \cdot u_{f}$ with $h \in L^{p}(\Omega)$ then $\frac{h}{N(f)}$ is $\mathcal{F}_{f}$-measurable and for every $\varphi \in L_{\infty}(\Omega, \Sigma, \mu)$ we have

$$
\mathbb{E}^{g}(\varphi) \cdot g=P\left(\varphi h \cdot u_{f}\right)=N(f) \mathbb{E}^{f}\left(\frac{\varphi \cdot h}{N(f)}\right) \cdot u_{f}=h \mathbb{E}^{f}(\varphi) \cdot u_{f}=\mathbb{E}^{f}(\varphi) \cdot g
$$

Hence

$$
\mathbb{E}^{g}(\varphi)=\mathbf{1}_{\mathbf{V S}(g)} \cdot \mathbb{E}^{f}(\varphi)=\mathbf{1}_{\text {Supp } h} \mathbb{E}^{f}(\varphi) .
$$

Let now $g$ be a general element of $R(P)$. For every $\varphi \in L_{\infty}(\Omega)$ the equation

$$
P(\varphi \cdot(f+g))=P(\varphi \cdot f)+P(\varphi \cdot g)
$$

is equivalent to

$$
\mathbb{E}^{f+g}(\varphi) \cdot(f+g)=\mathbb{E}^{f}(\varphi) \cdot f+\mathbb{E}^{g}(\varphi) \cdot g
$$


Let $g=h \cdot u_{f}+g^{\prime}$ be the orthogonal decomposition, i.e. $h=\left\langle\left\langle g \mid u_{f}\right\rangle\right\rangle$ and $g^{\prime} \perp f$. Note that $h \cdot u_{f}=E_{f} g \in R(P)$. Set $A=\mathbf{V S}(f), B=\mathbf{V S}(g)$ and $B^{\prime}=\mathbf{V S}\left(g^{\prime}\right)$. Taking the images of both sides of (17) by the orthogonal projection $I-E_{f}$ we obtain

$$
\mathbb{E}^{f+g}(\varphi) \cdot g^{\prime}=\mathbb{E}^{g}(\varphi) \cdot g^{\prime}
$$

hence $\mathbf{1}_{B^{\prime}} \mathbb{E}^{f+g}(\varphi)=\mathbf{1}_{B^{\prime}} \mathbb{E}^{g}(\varphi)$. Then by (17) again, $\mathbf{1}_{B^{\prime}} \mathbb{E}^{f+g}(\varphi) f=\mathbf{1}_{B^{\prime}} \mathbb{E}^{f}(\varphi) f$ and finally

$$
\mathbf{1}_{A \cap B^{\prime}} \mathbb{E}^{f+g}(\varphi)=\mathbf{1}_{A \cap B^{\prime}} \mathbb{E}^{f}(\varphi)=\mathbf{1}_{A \cap B^{\prime}} \mathbb{E}^{g}(\varphi) .
$$

On the other hand similarly to (17) we have

$$
\mathbb{E}^{h \cdot u_{f}-g}(\varphi) \cdot\left(h \cdot u_{f}-g\right)=\mathbb{E}^{h u_{f}}(\varphi) \cdot h u_{f}-\mathbb{E}^{g}(\varphi) \cdot g .
$$

Since $h \cdot u_{f}-g=-g^{\prime}$ we deduce that

$$
\mathbf{1}_{\Omega \backslash B^{\prime}} \mathbb{E}^{h u_{f}}(\varphi) \cdot h u_{f}=\mathbf{1}_{\Omega \backslash B^{\prime}} \mathbb{E}^{g}(\varphi) \cdot g,
$$

hence

$$
\mathbf{1}_{B \backslash B^{\prime}} \mathbb{E}^{h u_{f}}(\varphi)=\mathbf{1}_{B \backslash B^{\prime}} \mathbb{E}^{g}(\varphi) .
$$

We have $\mathbb{E}^{h u_{f}}(\varphi)=\mathbf{1}_{\text {Supp } h} \mathbb{E}^{f}(\varphi)$ by eq. (16). Hence since $B \backslash B^{\prime} \subset \operatorname{Supp} h$, eq. (19) gives

$$
\mathbf{1}_{B \backslash B^{\prime}} \mathbb{E}^{f}(\varphi)=\mathbf{1}_{B \backslash B^{\prime}} \mathbb{E}^{g}(\varphi)
$$

which together with eq. (18) gives

$$
\mathbf{1}_{A \cap B} \mathbb{E}^{f}(\varphi)=\mathbf{1}_{A \cap B} \mathbb{E}^{g}(\varphi)
$$

for every $\varphi \in L_{\infty}(\Omega, \Sigma, \mu)$. In particular

$$
\begin{aligned}
\mathbf{1}_{\mathbf{V S}(f) \cap \mathbf{V S}(g)} & =\mathbf{1}_{\mathbf{V S}(f) \cap \mathbf{V S}(g)} \mathbb{E}^{g}\left(\mathbf{1}_{\mathbf{V S}(g)}\right) \\
& =\mathbf{1}_{\mathbf{V S}(f) \cap \mathbf{V S}(g)} \mathbb{E}^{f}\left(\mathbf{1}_{\mathbf{V S}(g)}\right) \\
& =\mathbf{1}_{\mathbf{V S}(f) \cap \mathbf{V S}(g)} \mathbb{E}^{f}\left(\mathbf{1}_{\mathbf{V S}(f) \cap \mathbf{V S}(g)}\right),
\end{aligned}
$$

hence

$$
\mathbb{E}^{f}\left(\mathbf{1}_{\mathbf{V S}(f) \cap \mathbf{V S}(g)}\right) \geq \mathbf{1}_{\mathbf{V S}(f) \cap \mathbf{V S}(g)}
$$

and since $\mathbb{E}^{f}$ is a contraction in $L_{p}\left(\Omega, \Sigma, N(f)^{p} \cdot \mu\right)$ we have in fact

$$
\mathbb{E}^{f}\left(\mathbf{1}_{\mathbf{V S}(f) \cap \mathbf{V S}(g)}\right)=\mathbf{1}_{\mathbf{V S}(f) \cap \mathbf{V S}(g)},
$$

that is, $\mathbf{V S}(f) \cap \mathbf{V S}(g) \in \mathcal{F}_{f}$. In particular $\mathbf{1}_{\mathbf{V S}(g)} \cdot f=\mathbf{1}_{\mathbf{V S}(f) \cap \mathbf{V S}(g)} \cdot f \in R(P)$. More generally for every $A \in \mathcal{F}_{g}$ its trace $\operatorname{VS}(f) \cap A$ belongs to $\mathcal{F}_{f}$ (as is easily seen by treating separately the cases $A \subset \mathbf{V S}(g)$ and $A=\Omega \backslash \mathbf{V S}(g))$. Let $\mathcal{F}$ be the $\sigma$-algebra consisting of sets $A \in \Sigma$ such that $A \cap \mathbf{V S}(f)$ belongs to $\mathcal{F}_{f}$ for every $f \in R(P)$. Then for every $f \in R(P)$ and $\varphi \in L_{0}(\Omega, \Sigma, \mu)$ the function $\mathbf{1}_{\mathbf{V S}(f)} \varphi$ is $\mathcal{F}$ measurable iff it is $\mathcal{F}_{f}$-measurable, and the Lemma follows. 
Lemma 4.3. There is a weight $w \in L_{0}(\Omega, \Sigma, \mu)$ with support $\mathbf{V S}(R(P))$ such that for every $f \in R(P), w^{-1} N(f)$ is $\mathcal{F}$-measurable.

Proof. a) First we claim that for every $f, g \in R(P)$ then $\mathbf{1}_{\mathbf{V S}(f)} \frac{N(g)}{N(f)}$ is $\mathcal{F}$-measurable. Since $E_{f} g=\left\langle\left\langle g \mid u_{f}\right\rangle\right\rangle u_{f} \in R(P)$ by Lemma 2.2, it results from Lemma 4.2 that $N(f)^{-1}\left\langle\left\langle g \mid u_{f}\right\rangle\right\rangle=N(f)^{-2}\langle\langle g \mid f\rangle\rangle$ is $\mathcal{F}$-measurable; hence its absolute value $N(f)^{-2}$ $|\langle\langle g \mid f\rangle\rangle|$ is $\mathcal{F}$-measurable, and similarly $N(g)^{-2}|\langle\langle f \mid g\rangle\rangle|$ is $\mathcal{F}$-measurable too. Then the ratio of these functions, that is $\mathbf{1}_{\text {Supp }}\langle\langle g \mid f\rangle\rangle(g)^{2} N(f)^{-2}$ is $\mathcal{F}$-measurable, and so is its square root $\mathbf{1}_{\text {Supp }\langle\langle g \mid f\rangle\rangle} N(g) N(f)^{-1}$. Replacing $g$ by $g_{\varepsilon}=g+\varepsilon f, \varepsilon>0$ we obtain that $\mathbf{1}_{\text {Supp }\left\langle\left\langle g_{\varepsilon} \mid f\right\rangle\right\rangle} N\left(g_{\varepsilon}\right) N(f)^{-1}$ is $\mathcal{F}$ measurable. When $\varepsilon \rightarrow 0$ we have $g_{\varepsilon} \rightarrow g, N\left(g_{\varepsilon}\right) \rightarrow N(g)$ (in $L_{p}$-norm) and $\operatorname{Supp}\left\langle\left\langle g_{\varepsilon} \mid f\right\rangle\right\rangle \rightarrow \operatorname{Supp} N(f)=\mathbf{V S}(f)$ (in probability). At the limit $\mathbf{1}_{\mathbf{V S}(f)} \frac{N(g)}{N(f)}$ is $\mathcal{F}$-measurable.

b) Let $\left(f_{i}\right)_{i \in I}$ be a maximal family of non zero elements in $R(P)$ with pairwise almost disjoint functional supports $\mathbf{V S}\left(f_{i}\right)$. Then $\mathbf{V S}(R(P))=\bigvee_{i \in I} \mathbf{V S}\left(f_{i}\right)$ : if $f \in$ $R(P)$ then, since $S=\bigvee_{i \in I} \mathbf{V S}\left(f_{i}\right)$ belongs to $\mathcal{F}$, so does its complementary set $S^{c}$, and thus $\mathbf{1}_{S^{c}} f \in R(P)$; then, by maximality of the family $\left(f_{i}\right)$, we have $\mathbf{1}_{S^{c}} . f=0$, that is, $f=\mathbf{1}_{S} \cdot f$. We set $w=\sum_{i \in I} N\left(f_{i}\right)$ (which converges in $L_{0}(\Omega, \Sigma, \mu)$ ): this is a $\Sigma$-measurable weight with support $\mathbf{V S}(R(P))$. For every $f \in R(P)$ and every $i \in I, \mathbf{1}_{\mathbf{V S}\left(f_{i}\right)} w^{-1} N(f)=\mathbf{1}_{\mathbf{V S}\left(f_{i}\right)} N\left(f_{i}\right)^{-1} N(f)$ is $\mathcal{F}$-measurable; hence $w^{-1} N(f)=$ $\sum_{i \in I} \mathbf{1}_{\mathbf{V S}\left(f_{i}\right)} w^{-1} N(f)$ is $\mathcal{F}$-measurable.

We can now give the

Proof of the Thm. 0.1. Consider the new measure $\nu=w^{p} \cdot \mu$, which has support $\Omega_{P}=\mathbf{V S}(R(P))$ and set $T: L_{p}\left(\Omega_{P}, \Sigma_{P}, \mu\right) \rightarrow L_{p}\left(\Omega_{P}, \Sigma_{P}, \nu\right)$, defined by $T f=w^{-1} f$ (we denote by $\Sigma_{P}$ the trace of $\Sigma$ on $\Omega_{P}$ ). Then $T$ is an isometry; $Y:=\left(T \otimes \operatorname{Id}_{\mathrm{H}}\right)(R(P)$ ) is a $L_{\infty}\left(\mathcal{F}_{P}\right)$-module isometric to $R(P)$ and for every $f \in Y$ its new random norm $\widetilde{N}(f)=w^{-1} N(f)$ belongs to $L_{p}\left(\Omega_{P}, \mathcal{F}_{P}, \nu\right)$. It results from an argument in [13] that $Y$ is isometric to $\left(\bigoplus_{i \in I} L_{p}\left(\Omega_{i}, \mathcal{F}_{\mid \Omega_{i}}, \nu_{\mid \Omega_{i}} ; H_{i}\right)\right)_{\ell_{p}}$, for some families $\left(\Omega_{i}\right)$ of pairwise almost disjoint sets in $\mathcal{F}$ and $\left(H_{i}\right)$ of Hilbert spaces. Set then $\widehat{w}_{i}=\left(\mathbb{E}\left(\mathbf{1}_{\Omega_{i}} \cdot w^{p} \mid \mathcal{F}\right)\right)^{1 / p}$, and define an isometry $S_{i}: L_{p}\left(\Omega_{i}, \mathcal{F}_{\mid \Omega_{i}}, \nu_{\mid \Omega_{i}}\right) \rightarrow L_{p}\left(\Omega_{i}, \mathcal{F}_{\mid \Omega_{i}}, \mu_{\mid \Omega_{i}}\right)$ by $S_{i} f=\widehat{w}_{i} \cdot f$. Then each $S_{i} \otimes \operatorname{Id}_{\mathrm{H}_{\mathrm{i}}}$ is an onto isometry $L_{p}\left(\Omega_{i}, \mathcal{F}_{\mid \Omega_{i}}, \nu_{\mid \Omega_{i}} ; H_{i}\right) \rightarrow L_{p}\left(\Omega_{i}, \mathcal{F}_{\mid \Omega_{i}}, \mu_{\mid \Omega_{i}} ; H_{i}\right)$; the collection of these isometries induces an isometry of the corresponding $\ell_{p}$-direct sums. The proof of Thm. 0.1 is complete.

Let us finally adapt to the present situation the argument of [13] for the commodity of the reader (and for further reference in Section 5).

Lemma 4.4. Let $(\Omega, \Sigma, \nu)$ be a localizable measure space, $\mathcal{F}$ be a sub-sigma algebra such that $(\Omega, \mathcal{F}, \nu)$ is still localizable and $H$ be a Hilbert space. Let $Y$ be a closed $L_{\infty}(\mathcal{F})$-submodule of $L_{p}(\Omega, \Sigma, \nu ; H)$ such that for every $f \in Y$ its random norm $N(f)$ is $\mathcal{F}$-measurable. Then there exist a family $\left(\Omega_{i}\right)_{i \in I}$ of pairwise almost disjoint members of $\mathcal{F}$, a family $\left(\mathcal{H}_{i}\right)$ of Hilbert spaces (of lower Hilbertian dimension than $H$ ) and a random norm preserving isometry from $Y$ onto $\left(\bigoplus_{i \in I} L_{p}\left(\Omega_{i}, \mathcal{F}_{\mid \Omega_{i}}, \nu_{\mid \Omega_{i}} ; \mathcal{H}_{i}\right)\right)_{\ell_{p}}$. 
Proof. Note that by an elementary polarization argument all the scalar products $\left\langle\langle f \mid g\rangle, f, g \in Y\right.$ are $\mathcal{F}$-measurable. Hence for every $f \in Y$, the projection $E_{f}$ restricts to a projection from $Y$ onto $L_{p}(\Omega, \mathcal{F}) \cdot u_{f}$. It results that for every closed $L_{\infty}(\mathcal{F})$-submodule $Z$ of $Y$ there is an orthogonal projection from $Y$ onto $Z$ (which is the restriction of the orthogonal projection from $L_{p}(\Omega, \Sigma ; H)$ onto the closed $L_{\infty}(\Sigma)$ submodule generated by $Z)$. In particular $Y=Z \oplus\left(Z^{\perp} \cap Y\right)$.

Remark that if $A \in \mathcal{F}$ is $\nu$-sigma-finite and $M \subset Y$ is a closed $L_{\infty}(\mathcal{F})$-submodule such that $\mathbf{V S}(M) \supset A$ then there exists $g \in M$ such that $\mathbf{V S}(g)=A$ : take a maximal family $\left(g_{n}\right)$ in $M$ of norm-one elements with almost disjoint functional supports included in $A$; this family is necessarily at most countable and $\bigvee_{n} \mathbf{V S}\left(g_{n}\right)=A$; then set $g=\sum_{n} 2^{-n} g_{n}$.

Now we claim that for every $A \in \mathcal{F}, A \subset \mathbf{V S}(Y)$ with positive measure, there exists a $\mathcal{F}$-measurable subset $B$ of $A$ of positive measure and a family of pairwise orthogonal element $\left(f_{\gamma}\right)_{\alpha \in \Gamma_{B}}$, such that $\mathbf{V S}\left(f_{\gamma}\right)=B$ for every $\gamma \in \Gamma_{B}$, which generates $\mathbf{1}_{B} \cdot Y$ as closed $L_{\infty}(\mathcal{F})$-submodule. For, let $A^{\prime} \subset A$ be a sigma-finite $\mathcal{F}$-measurable subset with positive measure, and $\left(g_{\gamma}\right)_{\gamma \in \Gamma}$ be a maximal family of pairwise orthogonal elements of $Y$ with $\mathbf{V S}\left(g_{\gamma}\right)=A^{\prime}$. If this family generates $\mathbf{1}_{A^{\prime}} . Y$ as closed $L_{\infty}(\mathcal{F})$-submodule we can take $B=A^{\prime}$. If not, consider the set $M=\left\{f \in Y \mid f \perp g_{\gamma}, \forall \gamma \in \Gamma\right\}$. Then $M$ is a closed $L_{\infty}(\mathcal{F})$-submodule of $Y$, and $\mathbf{V S}(M) \not \supset A^{\prime}$ by the maximality of $\left(g_{\gamma}\right)_{\gamma \in \Gamma}$ (and the preceding remark). Let $B=A^{\prime} \backslash \mathbf{V S}(M)$, then $\left(\mathbf{1}_{B} g_{\gamma}\right)_{\gamma \in \Gamma}$ is a maximal family in $\mathbf{1}_{B} \cdot Y$ of nonzero, pairwise orthogonal elements of $\mathbf{1}_{B} \cdot Y$. Consequently it generates $\mathbf{1}_{B} \cdot Y$ as $L_{\infty}(\mathcal{F})$-submodule, and moreover $\mathbf{V S}\left(\mathbf{1}_{B} g_{\gamma}\right)=B$ for every $\gamma \in \Gamma$.

Let now $\left(\Omega_{i}\right)_{i \in I}$ be a maximal family of $\mathcal{F}$-measurable almost disjoint subsets of $\mathbf{V S}(Y)$ of positive measure, such that there exists for each $i \in I$ a family $\left(f_{\gamma}^{i}\right)_{\gamma \in \Gamma_{i}}$ of pairwise orthogonal elements with $\mathbf{V S}\left(f_{\gamma}^{i}\right)=\Omega_{i}$ for every $\gamma \in \Gamma_{i}$, which generates $\mathbf{1}_{\Omega_{i}} \cdot Y$ as closed $L_{\infty}(\mathcal{F})$-submodule. By the claim, we have $\bigvee_{i \in I} \Omega_{i}=\mathbf{V S}(Y)$. Every $f \in \mathbf{1}_{\Omega_{i}} \cdot Y$ can be written $f=\sum_{\gamma \in \Gamma_{i}} \varphi_{\gamma} f_{\gamma}^{i}$ with $\varphi_{\gamma} \in L_{0}\left(\left(\Omega_{i}, \mathcal{F}_{\mid \Omega_{i}}, \nu\right)\right.$; then $N(f)=\left(\sum_{\gamma \in \Gamma_{i}}\left|\varphi_{\gamma}\right|^{2} N\left(f_{\gamma}^{i}\right)^{2}\right)^{1 / 2} \in L_{p}\left(\Omega_{i}, \mathcal{F}_{\mid \Omega_{i}}, \nu\right)$.

Note that, by refining if necessary the "partition" $\left(\Omega_{i}\right)$ we may suppose that each $\Omega_{i}$ has finite $\nu$-measure. Then, replacing each $f_{\gamma}^{i}$ by $u_{f_{\gamma}^{i}}=N\left(f_{\gamma}^{i}\right)^{-1} f_{\gamma}^{i}$, we may assume that $N\left(f_{\gamma}^{i}\right)=\mathbf{1}_{\Omega_{i}}$. We have then $N(f)=\left(\sum_{\gamma \in \Gamma_{i}}\left|\varphi_{\gamma}\right|^{2}\right)^{1 / 2}$ for each $f=\sum_{\gamma \in \Gamma_{i}} \varphi_{\gamma} f_{\gamma}^{i}$ in $\mathbf{1}_{\Omega_{i}} \cdot Y$. Let $\mathcal{H}_{i}=\ell^{2}\left(\Gamma_{i}\right)$. Then $T_{i}: L_{p}\left(\Omega_{i}, \mathcal{F}_{\mid \Omega_{i}}, \nu ; \mathcal{H}_{i}\right) \rightarrow \mathbf{1}_{\Omega_{i}} \cdot Y$, $\sum_{\gamma \in \Gamma_{i}} \varphi_{\gamma} e_{\gamma} \mapsto \sum_{\gamma \in \Gamma_{i}} \varphi_{\gamma} u_{f_{\gamma}^{i}}$ is an (onto) isometry (preserving the random norm), and finally $Y$ is isometric to $\left(\bigoplus_{i \in I} L_{p}\left(\Omega_{i} ; \mathcal{H}_{i}\right)\right)_{\ell_{p}}$.

For proving the assertion about the Hilbertian dimension of $\mathcal{H}_{i}$, suppose that for some $i \in I$, the Hilbertian dimension $d_{H}$ of $H$ is strictly smaller than that of $\mathcal{H}_{i}, d_{\mathcal{H}_{i}}$. We distinguish two cases:

(i) if $H$ is finite dimensional: select a finite subset $\Gamma_{i}^{\prime}$ of $\Gamma_{i}$ with cardinality $d_{H}+1$; since $\left\langle\left\langle f_{\gamma}^{i} \mid f_{\delta}^{i}\right\rangle\right\rangle=0$ for every $\gamma \neq \delta \in \Gamma_{i}^{\prime}$, there exists $\omega \in \Omega$ such that $\left\langle\left\langle f_{\gamma}^{i} \mid f_{\delta}^{i}\right\rangle\right\rangle(\omega)=0$, i.e. the vectors $f_{\gamma}^{i}(\omega), \gamma \in \Gamma_{i}^{\prime}$ of $H$ are pairwise orthogonal: a 
contradiction.

(ii) if $H$ is infinite dimensional: for every $x \in H$ the set $\left\{\gamma \in \Gamma_{i}\left|\left\langle f_{\gamma}^{i} \mid \mathbf{1}_{\Omega_{i}} x\right\rangle\right\rangle \neq 0\right\}$ is at most countable (since $\sum_{\gamma} \mid\left\langle\left.\left\langle f_{\gamma}^{i} \mid \mathbf{1}_{\Omega_{i}} x\right\rangle\right|^{2} \leq N\left(\mathbf{1}_{\Omega_{i}} \cdot x\right)^{2}=\mathbf{1}_{\Omega_{i}}\|x\|^{2}\right.$ ), hence if $D$ is a dense set in $H$ of cardinality $d_{H}$, the set $\left\{\gamma \in \Gamma_{i} \mid \exists x \in D,\left\langle\left\langle f_{\gamma}^{i} \mid \mathbf{1}_{\Omega_{i}} x\right\rangle \neq \neq 0\right\}\right.$ has cardinality $d_{H}<d_{\mathcal{H}_{i}}=\# \Gamma_{i}$. Hence there exists some $\gamma \in \Gamma_{i}$ such that $f_{\gamma}^{i} \perp \mathbf{1}_{\Omega_{i}} x$ for every $x \in D$, and thus for every $x \in H$, which means $f_{\gamma}^{i}=0$, a contradiction.

Remark 4.5. The final argument in the proof of Lemma 4.4 shows indeed that if $L_{p}(\Omega, \Sigma, \nu ; \mathcal{H})$ embeds in $L_{p}(\Omega, \Sigma, \nu ; H)$ by a modularly isometric map then $\operatorname{dim} \mathcal{H} \leq$ $\operatorname{dim} H$.

Remark 4.6. In a forthcoming paper ([10]) it will be proved that contractively complemented sublattices of $L_{p}\left(L_{q}\right)$ are isometric to "abstract $L_{p}\left(L_{q}\right)$ spaces", i.e. bands in $L_{p}\left(L_{q}\right)$ spaces. Let us show how this permits to deduce shortly the essence of Thm. 0.1 from Lemma 4.1.

As in the proof of Lemma 4.1 let $\left(f_{\alpha}\right)_{\alpha \in \mathcal{A}}$ be a maximal family of non zero, pairwise orthogonal elements of $R(P)$ and $Z=\bigoplus_{\alpha} L_{p}(\Omega, \Sigma, \mu) \cdot u_{f_{\alpha}}$ be the closed $L_{\infty}(\Sigma)$-submodule generated by $R(P)$. There is clearly a $\Sigma$-modular isometry $U$ from the closed submodule $\mathrm{Z}$ onto a band $Y$ of the Banach lattice $L_{p}(\Omega, \Sigma, \mu ; \mathcal{H})$ where $\mathcal{H}$ is the discrete Banach lattice $\ell_{2}(A)$, such that $U e_{\alpha}=N\left(f_{\alpha}\right) e_{\alpha}$, where $\left(e_{\alpha}\right)_{\alpha \in A}$ is a Hilbertian basis of $\mathcal{H}$. Then $\left.P\right|_{Z}$ is similar by $U$ to a contractive projection $\widehat{P}$ of $Y$ which preserves the spaces $Y_{\alpha}=L_{p}\left(A_{\alpha}\right) \cdot e_{\alpha}$ (where $A_{\alpha}=\operatorname{Supp} N\left(f_{\alpha}\right)$ ) by Lemma 2.1 if $p=1$ and Cor. 3.6 if $p>1$, as well as the elements $N\left(f_{\alpha}\right) \cdot e_{\alpha}$. By the classical (scalar) theorem of Douglas if $p=1$, Andô if $p>1,\left.\widehat{P}\right|_{Y_{\alpha}}$ is positive and its image is a sublattice of $Y_{\alpha}$. Since $Y=\bigoplus_{\alpha} Y_{\alpha}$ is a decomposition in disjoint subbands, $\widehat{P}$ is itself positive and its range is a sublattice of $Y$, hence of $L_{p}(\mathcal{H})$. By the analysis of contractive projections on sublattices in $L_{p}\left(L_{q}\right)$-spaces developed in [10], the range $R(\widehat{P})$ is an abstract $L_{p}\left(L_{2}\right)$-space, hence by [13] it is Banach-isometric to a $\ell_{p}$-direct sum $\bigoplus_{i \in I} L_{p}\left(\Omega_{i}, H_{i}\right)$, where the $H_{i}$ are Hilbert spaces.

\section{Structure of the contractive projections}

Theorem 5.1. Let $1 \leq p<\infty, p \neq 2$. For every contractive projection $P$ of $L_{p}(H)$ there exist a family $\left(u_{\gamma}\right)_{\gamma \in \Gamma}$ of pairwise orthogonal elements of $L_{\infty}(H)$, a positive contractive projection $\widetilde{P}$ of $L_{p}(\Omega)$ and, if $p=1$, a contractive linear operator $V: L_{1}(H) \rightarrow L_{1}(H)$ verifying $\operatorname{ker} V \supset \mathbf{1}_{A} L_{1}(H)$ where $A=\bigvee_{\gamma \in \Gamma} \mathbf{V S}\left(u_{\gamma}\right)$, and $R(V) \subset \sum_{\gamma} R(\widetilde{P}) \cdot u_{\gamma}$, such that:

$$
P f= \begin{cases}\sum_{\gamma} \widetilde{P}\left(\left\langle\left\langle f \mid u_{\gamma}\right\rangle\right)\right) u_{\gamma} & \text { if } p \neq 1 \\ \sum_{\gamma} \widetilde{P}\left(\left\langle\left\langle f \mid u_{\gamma}\right\rangle\right)\right) u_{\gamma}+V(f) & \text { if } p=1\end{cases}
$$


for every $f \in L_{p}(H)$.

Conversely for every family $\left(u_{\gamma}\right)_{\gamma \in \Gamma}$ of pairwise orthogonal elements of $L_{\infty}(H)$, every positive contractive projection $\widetilde{P}$ of $L_{p}(\Omega)$ [and every linear contraction $V$ of $L_{1}(H)$ satisfying the previous conditions of kernel and range in the case $\left.p=1\right]$, the formula (20) defines a contractive projection $P$ of $L_{p}(H)$.

Moreover if $p \neq 1$ the inequality $N(P f) \leq \widetilde{P}(N(f))$ holds for every $f \in L_{p}(H)$ [this happens also for a contractive projection of $L_{1}(H)$ for which the operator $V$ of formula (20) is zero]. ( $\widetilde{P}$ is a "majorizing $L_{p}$-contraction" for $P$ in the terminology of $[8])$.

The proof of Thm. 5.1 will require the two following Lemmas, the first of which is specific to the $p=1$ case:

Lemma 5.2. Let $P$ be a contractive projection in $L_{1}(H)$. Then $P f=0$ for every $f \in L_{1}(H)$ with $\mathbf{V S}(f) \subset \mathbf{V S}(R(P))$ and $f \perp R(P)$.

Proof. Assume that $f \perp R(P)$ and $\mathbf{V S}(f) \subset \mathbf{V S}(h)$ for some $h \in R(P)$. Then $g:=P f+\mathbf{1}_{(\mathbf{V S}(P f))^{c}} h$ belongs to $R(P)$ and $\mathbf{V S}(g) \supset \mathbf{V S}(f) \cup \mathbf{V S}(P f)$. We have for every $t>0$ :

$$
\begin{aligned}
\int\left(N(g)^{2}+t^{2} N(f)^{2}\right)^{1 / 2} d \mu & =\|g+t f\| \\
& \geq\|P(g+t f)\|=\|g+t P f\| \\
& =(1+t)\|P f\|+\| \mathbf{1}_{(\mathbf{V S}(P f))^{c} \cdot h \|} \\
& =\|g\|+t\|P f\| .
\end{aligned}
$$

Hence:

$$
\|P f\| \leq \lim _{t \rightarrow 0}\left(\frac{\|g+t f\|-\|g\|}{t}\right)=\lim _{t \rightarrow 0} \int \frac{\left(N(g)^{2}+t^{2} N(f)^{2}\right)^{1 / 2}-N(g)}{t} d \mu=0 .
$$

Lemma 5.3. Let $P$ be a contractive projection in $L_{p}(H)$. There exists a positive contractive projection $\widetilde{P}$ on $L_{p}(\Omega, \Sigma, \mu)$ such that $P\left(\varphi \cdot u_{f}\right)=(\widetilde{P} \varphi) \cdot u_{f}$ for every $f \in R(P)$ and $\varphi \in L_{p}(\Omega, \Sigma, \mu)$.

Proof. Let $\mathcal{F}$ be the $\sigma$-algebra of Lemma 4.2 and $w$ be the weight of Lemma 4.3 . Define $\widetilde{P}_{f}(\varphi)$ as in the proof of Lemma 4.2. Recall that for every $f \in R(P)$ the function $w^{-1} N(f)$ is $\mathcal{F}$-measurable. We have then for every $h \in L_{\infty}(\Omega, \mathcal{F}, \mu)$ :

$$
\begin{aligned}
\int \widetilde{P}_{f}(\varphi) h N(f)^{p-1} d \mu & =\int \mathbb{E}_{N(f)^{p} \cdot \mu}^{\mathcal{F}}\left(N(f)^{-1} \mathbf{1}_{\mathbf{V S}(f)} \varphi\right) h N(f)^{p} \cdot d \mu \\
& =\int \mathbf{1}_{\mathbf{V S}(f)} \varphi \cdot h N(f)^{p-1} \cdot d \mu
\end{aligned}
$$




$$
\begin{aligned}
& =\int\left(\mathbf{1}_{\mathbf{V S}(R(P))} w^{-1} \varphi\right) \cdot\left(\mathbf{1}_{\mathbf{V S}(f)} h\left(w^{-1} N(f)\right)^{p-1}\right) w^{p} \cdot d \mu \\
& =\int \mathbb{E}_{w^{p} \mu}^{\mathcal{F}}\left(\mathbf{1}_{\mathbf{V S}(R(P))} w^{-1} \varphi\right) \mathbf{1}_{\mathbf{V S}(f)} h\left(w^{-1} N(f)\right)^{p-1} w^{p} \cdot d \mu \\
& =\int \mathbf{1}_{\mathbf{V S}(f)} w \mathbb{E}_{w^{p} \mu}^{\mathcal{F}}\left(\mathbf{1}_{\mathbf{V S}(R(P))} w^{-1} \varphi\right) h N(f)^{p-1} d \mu .
\end{aligned}
$$

Hence $\widetilde{P}_{f} \varphi=\mathbf{1}_{\mathbf{V S}(f)} \widetilde{P} \varphi$ if we set $\widetilde{P} \varphi=w \mathbb{E}_{w^{p} \cdot \mu}^{\mathcal{F}}\left(\mathbf{1}_{\mathbf{V S}(R(P))} w^{-1} \varphi\right)$ for every $\varphi \in$ $L_{p}(\Omega, \Sigma, \mu)$. Then $\widetilde{P}$ is a positive contractive projection in $L_{p}(\Omega, \Sigma, \mu)$ and $P\left(\varphi \cdot u_{f}\right)=$ $\widetilde{P}(\varphi) \cdot u_{f}$ for every $f \in R(P)$ and $\varphi \in L_{p}(\Omega, \Sigma, \mu)$.

Proof of Thm. 5.1. Let $Q$ be the orthogonal projection from $L_{p}(H)$ onto the closed submodule generated by $R(P)$. It results from Lemma 4.1 that if $\left(f_{\gamma}\right)_{\gamma \in \Gamma}$ is a maximal family of pairwise orthogonal elements of $R(P)$ then $Q=\sum_{\gamma \in \Gamma} E_{f_{\gamma}}$ (convergence for s.o.t.), hence $P Q=\sum_{\gamma \in \Gamma} P E_{f_{\gamma}}$. If $p>1$ we know by Cor. 3.6 that $E_{f_{\gamma}} P=P E_{f_{\gamma}}$ for every $\gamma$, hence $P=Q P=P Q$. If $p=1$ let $\Pi: L_{1}(H) \rightarrow L_{1}(H)$ the projection defined by $\Pi f=1_{\mathrm{VS}(R(P))} \cdot f$, then $\Pi$ and $I-\Pi$ are contractive. We have $Q \Pi=\Pi Q=Q$ and it results from the preceding Lemma 5.2 that $P(I-Q) \Pi=0$. Hence $P=P Q+V$, where $V=P(I-\Pi)$.

Let us express now $P E_{f}$ when $f \in R(P)$. If $\widetilde{P}$ is the positive projection in $L_{p}(\Omega, \Sigma, \mu)$ defined in Lemma 5.3 we have for every $g \in L_{p}(\Omega, \Sigma, \mu ; H)$

$$
P E_{f} g=P\left(\left\langle\left\langle g \mid u_{f}\right\rangle\right\rangle \cdot u_{f}\right)=\widetilde{P}\left(\left\langle\left\langle g \mid u_{f}\right\rangle\right\rangle\right) \cdot u_{f}
$$

The formula (20) in Thm. 5.1 is now clear if we set $u_{\gamma}=u_{f_{\gamma}}$.

Conversely given $\left(u_{\gamma}\right), \widetilde{P}$ and $V$, let us prove first that $P$ is a contraction. We have for every finite subset $G$ of $\Gamma$ (using the positivity of $\widetilde{P}$ ):

$$
\begin{aligned}
N\left(\sum_{\gamma \in G} \widetilde{P}\left(\left\langle\left\langle f \mid u_{\gamma}\right\rangle\right)\right) u_{\gamma}\right) & =\left(\sum_{\gamma \in G}\left|\widetilde{P}\left(\left\langle\left\langle f \mid u_{\gamma}\right\rangle\right\rangle\right)\right|^{2}\right)^{1 / 2} \\
& =\bigvee\left\{\left.\left|\sum_{\gamma \in G} a_{\gamma} \widetilde{P}\left\langle\left\langle f \mid u_{\gamma}\right\rangle\right\rangle\right|\left|a_{\gamma} \in \mathbb{C}, \sum_{\gamma \in G}\right| a_{\gamma}\right|^{2} \leq 1\right\} \\
& \leq \widetilde{P}\left(\bigvee\left\{\left.\left|\sum_{\gamma \in G} a_{\gamma}\left\langle\left\langle f \mid u_{\gamma}\right\rangle\right\rangle\right|\left|a_{\gamma} \in \mathbb{C}, \sum_{\gamma \in G}\right| a_{\gamma}\right|^{2} \leq 1\right\}\right) \\
& =\widetilde{P}\left(\left(\sum_{\gamma \in G}\left|\left\langle\left\langle f \mid u_{\gamma}\right\rangle\right\rangle\right|^{2}\right)^{1 / 2}\right) .
\end{aligned}
$$

Hence $\left.\| \sum_{\gamma \in G} \widetilde{P}\left(\left\langle f \mid u_{\gamma}\right\rangle\right\rangle\right) u_{\gamma} \|^{p} \leq \int\left(\sum_{\gamma \in G}\left|\left\langle\left\langle f \mid u_{\gamma}\right\rangle\right\rangle\right|^{2}\right)^{p / 2} d \mu$ and the sum $P_{0} f:=$ $\sum_{\gamma \in \Gamma} \widetilde{P}\left(\left\langle\left\langle f \mid u_{\gamma}\right\rangle\right\rangle\right) u_{\gamma}$ converges in $L_{p}(H)$. Moreover

$$
N\left(P_{0} f\right) \leq \widetilde{P}\left(\left(\left.\sum_{\gamma \in \Gamma}\left|\left\langle f \mid u_{\gamma}\right\rangle\right\rangle\right|^{2}\right)^{1 / 2}\right) \leq \widetilde{P}\left(N\left(\mathbf{1}_{A} \cdot f\right)\right)
$$


(see section 1.2 and the proof of Lemma 1.1) and

$$
\left\|P_{0} f\right\| \leq\left\|\mathbf{1}_{A} \cdot f\right\|
$$

where $A=\bigvee_{\gamma} \mathbf{V S}\left(u_{\gamma}\right)$. That $P_{0}$ is a projection follows immediately from the fact that $\widetilde{P}$ is. If $p=1$ we have to care with the contraction $V$. Since $\|V f\| \leq\left\|\mathbf{1}_{A^{c}} \cdot f\right\|$ we obtain $\|P f\| \leq\left\|\mathbf{1}_{A} \cdot f\right\|+\left\|\mathbf{1}_{A^{c}} \cdot f\right\|=\|f\|$. Then since $V P_{0}=0, P_{0} V=V$, it follows clearly that $P=P_{0}+V$ is a projection.

We can now give the structure theorem for contractive projections:

Theorem 5.4. For every contractive projection $P$ of $L_{p}(\Omega, \Sigma, \mu ; H)(1 \leq p<\infty$, $p \neq 2$ ) there exist:

- a modularly isometric automorphism $W$ of $L_{p}(H)$;

- a family $\left(\Omega_{i}\right)_{i \in I}$ of pairwise almost disjoint $\Sigma$-measurable subsets of $\Omega$ of positive measure;

- a family $\left(\mathcal{H}_{i}\right)_{i \in I}$ of Hilbert spaces;

- for every $i \in I$ a (strong operator) measurable family $\left(U_{i, \omega}\right)_{\omega \in \Omega}$ of isometric embeddings of $\mathcal{H}_{i}$ into $H$;

- a positive contractive projection $\widetilde{P}$ of $L_{p}(\Omega, \Sigma, \mu)$ commuting with the band projections associated with the sets $\Omega_{i}$;

- and if $p=1$ a contraction $V$ from $L_{1}\left(S, \Sigma_{\mid S}, \mu_{\mid S} ; H\right)$ into $R(P)$, where $S=$ $\Omega \backslash \bigvee_{i} \Omega_{i}$

such that (setting $V=0$ if $p>1)$ :

$$
P=W U\left(\sum_{i} \widetilde{P} M_{\Omega_{i}} \otimes \operatorname{Id}_{\mathcal{H}_{i}}\right) U^{\sharp} W^{-1}+V
$$

where $M_{\Omega_{i}}$ denotes the multiplication operator by the characteristic function $\mathbf{1}_{\Omega_{i}}$; $U$ is the modularly isometric embedding of $\bigoplus L_{p}\left(\Omega_{i}, \Sigma_{\mid \Omega_{i}}, \mu_{\mid \Omega_{i}} ; \mathcal{H}_{i}\right)$ into $L_{p}(H)$ naturally associated with the family $\left(U_{i, \omega}\right)_{i \in I, \omega \in \Omega}$ by mean of the formula:

$$
(U f)(\omega)=U_{i, \omega}(f(\omega)) \quad \text { when } \omega \in \Omega_{i}
$$

and similarly $U^{\sharp}: L_{p}(H) \rightarrow \bigoplus L_{p}\left(\Omega_{i}, \Sigma_{\mid \Omega_{i}}, \mu_{\mid \Omega_{i}} ; \mathcal{H}_{i}\right)$ is the modularly contractive map associated with the family $\left(U_{i, \omega}^{*}\right)_{i \in I, \omega \in \Omega}$.

Remark 5.5. In fact the families $\left(U_{i, \omega}\right)_{\omega \in \Omega}$ may be chosen locally constant, i.e. there is a partition of $\Omega_{i}$ in $\Sigma$-measurable subsets of positive measure on which $U_{i, \omega}$ is constant. 
Remark 5.6. In the case where $H$ is separable, it is a standard (and easy) fact that every modularly isometric automorphism $W$ of $L_{p}(H)$ is associated with a measurable family $\left(W_{\omega}\right)_{\omega \in \Omega}$ of unitary operators on $H$; so we recover the Theorem 0.3 of the Introduction.

Proof. By the proof of Thm. 0.1 in Section 4, there are a sub- $\sigma$ algebra $\mathcal{F}$ of $\Sigma$, a family $\left(\Omega_{i}\right)_{i \in I}$ of pairwise almost disjoint elements of $\mathcal{F}$, a positive weight $w$ on $\Omega$ with support $\bigvee_{i \in I} \Omega_{i}$, a family $\left(\mathcal{H}_{i}\right)_{i \in I}$ of Hilbert spaces and for every $i \in I$ an isometry $T_{i}$ from $L_{p}\left(\Omega_{i}, \mathcal{F}_{\mid \Omega_{i}},\left.\nu\right|_{\Omega_{i}} ; \mathcal{H}_{i}\right)$ into $L_{p}\left(\Omega_{i}, \mathcal{F}_{\mid \Omega_{i}}, \nu_{\mid \Omega_{i}} ; H\right)$ such that $R(P)=\bigoplus_{i \in I} w \cdot R\left(T_{i}\right)$ and moreover $N\left(T_{i} f\right)=N(f)$ for all $f \in L_{p}\left(\Omega_{i}, \mathcal{F}_{\mid \Omega_{i}}, \nu_{\mid \Omega_{i}} ; \mathcal{H}_{i}\right)$ (recall that $\left.\nu=w^{p} \cdot \mu\right)$. Moreover $P$ commutes with the action of $L_{\infty}(\mathcal{F})$, in particular with the multiplication operators $M_{\Omega_{i}}$.

Each $T_{i}$ extends uniquely to a modularly isometric map $\widetilde{T}_{i}$ from $L_{p}\left(\Omega_{i}, \Sigma_{\mid \Omega_{i}}, \nu_{\mid \Omega_{i}} ;\right.$ $\left.\mathcal{H}_{i}\right)$ onto the closed $L_{\infty}(\Sigma)$-submodule generated by $R\left(T_{i}\right)$ in $L_{p}(\Omega, \Sigma, \nu ; H)$ : set simply $\widetilde{T}_{i}\left(\sum_{k} \varphi_{k} f_{k}\right)=\sum_{k} \varphi_{k} T_{i}\left(f_{k}\right)$ when $\varphi_{1}, \ldots, \varphi_{n} \in L_{\infty}\left(\Omega_{i}, \Sigma_{\mid \Omega_{i}}\right)$ and $f_{1}, \ldots, f_{k} \in$ $L_{p}\left(\Omega_{i}, \mathcal{F}_{\mid \Omega_{i}}, \nu_{\mid \Omega_{i}} ; \mathcal{H}_{i}\right)$ and verify that $N\left(\sum_{k} \varphi_{k} T_{i}\left(f_{k}\right)\right)=N\left(\sum_{k} \varphi_{k} f_{k}\right)$ (since $T_{i}$ preserves the random scalar products).

Now define $S_{i}: L_{p}\left(\Omega_{i}, \Sigma_{\mid \Omega_{i}}, \mu_{\mid \Omega_{i}} ; \mathcal{H}_{i}\right) \rightarrow L_{p}(\Omega, \Sigma, \mu ; H)$ by $S_{i} f=w \widetilde{T}_{i}\left(w^{-1} f\right):$ the range $R\left(S_{i}\right)=w R\left(\widetilde{T}_{i}\right)$ is exactly $\mathbf{1}_{\Omega_{i}} \cdot Z$, where $Z$ is the closed $L_{\infty}(\Sigma)$-submodule generated by $R(P)$. We can glue up the maps $S_{i}$ and obtain a modularly isometric embedding $S$ from $\bigoplus_{i \in I} L_{p}\left(\Omega_{i}, \Sigma_{\mid \Omega_{i}}, \mu_{\mid \Omega_{i}} ; \mathcal{H}_{i}\right)$ into $L_{p}(\Omega, \Sigma, \mu ; H)$, with range $R(S)=Z$.

By Lemma 5.3 there exists a positive projection $\widetilde{P}$ on $L_{p}(\Omega, \Sigma, \mu)$ such that $P\left(\varphi \cdot u_{f}\right)=(\widetilde{P} \varphi) \cdot u_{f}$ for every $f \in R(P)$ and $\varphi \in L_{p}(\Omega, \Sigma, \mu)$. Note that $\widetilde{P}$ is $\mathcal{F}$ modular, in particular it commutes with every multiplication operator $M_{\Omega_{i}}, i \in I$.

If $A \in \mathcal{F}$ is a $\nu$-integrable subset of $\Omega_{i}$ and $e \in H_{i}$ we have $S_{i}\left(\mathbf{1}_{A} w \otimes e\right)=$ $w T_{i}\left(\mathbf{1}_{A} \otimes e\right) \in R(P)$, and $N\left(S_{i}\left(\mathbf{1}_{A} \cdot w \otimes e\right)\right)=N\left(\mathbf{1}_{A} \cdot w \otimes e\right)=\mathbf{1}_{A} \cdot w$, and consequently for $f=S_{i}\left(\mathbf{1}_{A} w \otimes e\right)$ we have $f=w \cdot u_{f}$. Thus for every $\psi \in L_{\infty}(\Omega, \Sigma, \mu) \cap L_{p}(\Omega, \Sigma, \mu)$ we have

$$
\begin{aligned}
P S_{i}\left(\psi \mathbf{1}_{A} w \otimes e\right) & =P\left(\psi \cdot w u_{f}\right)=\widetilde{P}\left(\psi \cdot w \mathbf{1}_{\Omega_{i}}\right) \cdot u_{f}=\widetilde{P}\left(\psi \cdot w \mathbf{1}_{\Omega_{i}}\right) \cdot w^{-1} S_{i}\left(\mathbf{1}_{A} w \otimes e\right) \\
& =S_{i}\left(\widetilde{P}\left(\psi \cdot w \mathbf{1}_{\Omega_{i}}\right) \cdot w^{-1} \cdot \mathbf{1}_{A} w \otimes e\right)=S_{i}\left(\widetilde{P}\left(\psi \cdot w \mathbf{1}_{\Omega_{i}}\right) \mathbf{1}_{A} \otimes e\right)
\end{aligned}
$$

hence by linearity and density we have for every $\varphi \in L_{p}\left(\Omega_{i}, \Sigma_{\mid \Omega_{i}}, \mu_{\mid \Omega_{i}}\right)$ and $e \in \mathcal{H}_{i}$ :

$$
P S_{i}(\varphi \otimes e)=S_{i}(\widetilde{P}(\varphi) \otimes e)
$$

that is, the restriction of $P$ to $\mathbf{1}_{\Omega_{i}} \cdot Z$ is similar by $S_{i}$ to the projection $\widetilde{P} \otimes \mathrm{id} \mathcal{H}_{i}$; consequently the restriction of $P$ to $Z$ is similar by $S$ to the projection $\sum_{i \in I} \widetilde{P} M_{\Omega_{i}} \otimes$ $\operatorname{id}_{\mathcal{H}_{i}}$.

In the case where $Z=L_{p}(\Omega, \Sigma, \mu ; H)$ we have necessarily $\operatorname{dim} H=\operatorname{dim} \mathcal{H}_{i}$ for every $i \in I$ since $S_{i}$ is a modularly isometric map from $L_{p}\left(\Omega_{i} ; \mathcal{H}_{i}\right)$ onto $\mathbf{1}_{\Omega_{i}} . Z=$ 
$L_{p}\left(\Omega_{i} ; H\right)$ (see Remark 4.5). Thus we may assume that $\mathcal{H}_{i}=H$ and the conclusion of Thm. 5.4 is obtained with $W=S$ and $U=\mathrm{Id}$.

In the general case we apply Lemma 4.4 to the $L_{\infty}(\Sigma)$ submodule $Z^{\perp}$. We find a family $\left(\Omega_{j}^{\prime}\right)_{j \in J}$ of pairwise almost disjoint members of $\Sigma$, a family $\left(\mathcal{K}_{j}\right)_{j \in J}$ of Hilbert spaces and a modularly isometric map $S^{\prime}$ from $\left(\bigoplus_{j \in J} L_{p}\left(\Omega_{j}^{\prime}, \Sigma_{\mid \Omega_{j}^{\prime}}, \mu_{\mid \Omega_{j}^{\prime}} ; \mathcal{K}_{j}\right)\right)_{\ell_{p}}$ onto $Z^{\perp}$. Note that now the sets $\Omega_{j}^{\prime}$ have no reason to belong to the smaller $\sigma$-algebra $\mathcal{F}$. We have $\bigvee_{j} \Omega_{j}^{\prime}=\mathbf{V S}\left(Z^{\perp}\right)$. For the commodity of the notation we may assume $\bigvee_{j} \Omega_{j}^{\prime}=\Omega$, adding if necessary one extra set $\Omega_{0}^{\prime}=\Omega \backslash \bigvee_{j} \Omega_{j}^{\prime}$ for which we set $\mathcal{K}_{0}=\{0\}$, the 0 -dimensional Hilbert space. Similarly, up to the cost of adding one extra set $\Omega_{0}=\Omega \backslash \Omega_{P}$ and setting $\mathcal{H}_{0}=\{0\}$, we may assume that $\bigvee_{i} \Omega_{i}=\Omega$. We may also refine the partition $\left(\Omega_{j}^{\prime}\right)$ by setting $\Omega_{i j}^{\prime}=\Omega_{i} \cap \Omega_{j}^{\prime}$ and removing the $\Omega_{i j}^{\prime}$ which are almost void. This operation gives a doubly indexed family $\left(\Omega_{i j}^{\prime}\right)_{i \in I ; j \in J_{i}}$.

For every $i \in I, j \in J_{i}$, set $L_{i j}=\mathcal{H}_{i} \oplus \mathcal{K}_{j}$ (direct Hilbertian sum). Then $L_{p}\left(\Omega_{i j}^{\prime} ; \mathcal{H}_{i}\right)$ and $L_{p}\left(\Omega_{i j}^{\prime} ; \mathcal{K}_{j}\right)$ identify naturally to a pair of mutually orthogonal $L_{\infty}(\Sigma)$-submodules of $L_{p}\left(\Omega_{i j}^{\prime} ; L_{i j}\right)$ : if $u_{i j}^{0}$ and $u_{i j}^{\prime 0}$ are the inclusion maps of $\mathcal{H}_{i}$, resp. $\mathcal{K}_{j}$ into $L_{i j}$ then $U_{i j}^{0}=\mathrm{id} \otimes u_{i j}^{0}$ and $U_{i j}^{\prime 0}=\mathrm{id} \otimes u_{i j}^{\prime 0}$ are the corresponding embeddings of $L_{p}\left(\Omega_{i j}^{\prime} ; \mathcal{H}_{i}\right)$ and $L_{p}\left(\Omega_{i j}^{\prime} ; \mathcal{K}_{j}\right)$ into $L_{p}\left(\Omega_{i j}^{\prime} ; L_{i j}\right)$. Since $u_{i j}^{0 *}$ and $u_{i j}^{\prime 0 *}$ are the orthogonal projections $L_{i j} \rightarrow \mathcal{H}_{i}$, resp. $L_{i j} \rightarrow \mathcal{K}_{j}$, we see that $U_{i j}^{0 \sharp}$ and $U_{i j}^{\prime 0 \sharp}$ are the orthogonal projections (in the sense given in Section 1.2) onto $L_{p}\left(\Omega_{i j}^{\prime} ; \mathcal{H}_{i}\right)$, resp. $L_{p}\left(\Omega_{i j}^{\prime} ; \mathcal{K}_{j}\right)$. have

Now define $W_{i j}^{0}: L_{p}\left(\Omega_{i j}^{\prime} ; L_{i j}\right) \rightarrow L_{p}\left(\Omega_{i j}^{\prime} ; H\right)$ by $W_{i j}^{0} f=S_{i}\left(U_{i j}^{0 \sharp} f\right)+S_{j}^{\prime}\left(U_{i j}^{\prime 0 \sharp} f\right)$ : we

$$
N\left(W_{i j}^{0} f\right)^{2}=N\left(S_{i}\left(U_{i j}^{0 \sharp} f\right)\right)^{2}+N\left(S_{i}^{\prime}\left(U_{i j}^{0 \sharp} f\right)\right)^{2}=N\left(U_{i j}^{0 \sharp} f\right)^{2}+N\left(U_{i j}^{\prime 0 \sharp} f\right)^{2}=N(f)^{2}
$$

since $S_{i}$ and $S_{i}^{\prime}$ are modularly isometric and have values in orthogonal subspaces $Z$, resp. $Z^{\perp}$. Hence $W_{i j}^{0}$ is modularly isometric and $R\left(W_{i j}^{0}\right)=\mathbf{1}_{\Omega_{i j}^{\prime}} Z+\mathbf{1}_{\Omega_{i j}^{\prime}} Z^{\perp}=$ $L_{p}\left(\Omega_{i j}^{\prime} ; H\right)$.

We know by the proof of Thm. 5.1 that $P=P Q+V$, where $Q$ is the orthogonal projection onto $Z$. Since $V$ satisfies the requirements of the theorem, we look only for a representation of $P_{0}=P Q$. From the first part of the proof we know that $P_{0} S_{i}=S_{i}\left(\widetilde{P} \otimes \mathrm{id}_{\mathcal{H}_{i}}\right)$; on the other hand $P_{0} S_{j}^{\prime}=0$ since $R\left(S_{j}^{\prime}\right) \subset Z^{\perp}=\operatorname{ker} Q$. Hence, for every $f \in L_{p}\left(\Omega_{i j} ; L_{i j}\right)$,

$$
P_{0} W_{i j}^{0} f=P_{0} S_{i} U_{i j}^{0 \sharp} f+P_{0} S_{j}^{\prime} U_{i j}^{\prime 0 \sharp} f=S_{i}\left(\widetilde{P} \otimes \operatorname{id}_{\mathcal{H}_{i}}\right) U_{i j}^{0 \sharp} f=W_{i j}^{0} U_{i j}^{0}\left(\widetilde{P} \otimes \operatorname{id}_{\mathcal{H}_{i}}\right) U_{i j}^{0 \sharp} f
$$

i.e. $P_{0}$ is similar by $W_{i j}^{0}$ to $U_{i j}^{0}\left(\widetilde{P} M_{\Omega_{i j}^{\prime}} \otimes \mathrm{id}_{\mathcal{H}_{i}}\right) U_{i j}^{0 \sharp}$.

Since $L_{p}\left(\Omega_{i j} ; L_{i j}\right)$ is modularly isometric to $L_{p}\left(\Omega_{i j} ; H\right)$ (by $\left.W_{i j}^{0}\right)$, we have $\operatorname{dim} L_{i j}=$ $\operatorname{dim} H$ by Rem. 4.5, so we may identify $L_{i j}$ with $H$ by an isomorphism $\theta_{i j}$. This isomorphism induces in turn a modular isometry $\Theta_{i j}=\operatorname{Id} \otimes \theta_{i j}$ from $L_{p}\left(\Omega_{i j}^{\prime} ; L_{i j}\right)$ onto $L_{p}\left(\Omega_{i j}^{\prime} ; H\right)$. Set $W_{i j}=W_{i j}^{0} \Theta_{i j}^{-1}$ : then $W_{i j}$ is a modular automorphism of $L_{p}\left(\Omega_{i j}^{\prime} ; H\right)$. Let also $u_{i j}=\theta_{i j} \circ u_{i j}^{0}$ be the embedding of $\mathcal{H}_{i}$ into $H$ resulting from this identification 
and $U_{i j}=\operatorname{id}_{L_{p}\left(\Omega_{i j}\right)} \otimes u_{i j}=\Theta_{i j} U_{i j}^{0}$ be the associated embedding of $L_{p}\left(\Omega_{i j} ; \mathcal{H}_{i}\right)$ into $L_{p}\left(\Omega_{i j} ; \mathcal{H}\right)$. Since $\Theta_{i j}^{-1}=\Theta_{i j}^{\sharp}$ we see that $P_{0}$ is similar by $W_{i j}$ to $U_{i j}\left(\widetilde{P} M_{\Omega_{i j}^{\prime}} \otimes \operatorname{id}_{\mathcal{H}_{i}}\right) U_{i j}^{\sharp}$.

Finally we glue up the automorphisms $W_{i j}$ to an automorphism $W$ of $L_{p}(\Omega ; \mu ; H)$ by setting

$$
W f=\sum_{i \in I} \sum_{j \in J_{i}} W_{i j} M_{\Omega_{i j}^{\prime}} f
$$

and similarly we glue up the embeddings $U_{i j}$ to an embedding $U$ of $\bigoplus_{i \in I} L_{p}\left(\Omega_{i} ; \mathcal{H}_{i}\right)$ into $L_{p}(\Omega ; H)$. The maps $W$ and $U$ are still modularly isometric and $P_{0}$ is similar by $W$ to $U\left(\sum_{i \in I} \widetilde{P} M_{\Omega_{i}} \otimes \operatorname{id}_{\mathcal{H}_{i}}\right) U^{\sharp}$.

\section{Annex: a proof of Corollary 3.6 specific to the complex case}

The following proof is an adaptation of that of Thm. 4.1 in [2]. We assume that $2<p<\infty$ (the case $1<p<2$ follows by duality).

If $f \in R(P)$ we introduce besides the projection $E_{f}$ (defined in $\S 1$ ) the operators $F_{f}$ and $G_{f}$ defined by

$$
F_{f} g=\mathbf{1}_{\mathbf{V S}(f)^{c}} g ; G_{f} g=\mathbf{1}_{\mathbf{V S}(f)} g-E_{f} g .
$$

Then $E_{f}, G_{f}$ and $F_{f}$ are commuting modularly contractive projections in $L_{p}(H)$ with $E_{f}+F_{f}+G_{f}=I$.

Let $f, g \in R(P)$, then the elements $A(f, g)$ and $B(f, g)$ defined in $\S 3$ (eqs. (5) and (5bis) belong to the range of $P^{*}$; so do the sum and difference: $M_{f}(g):=A(f, g)+$ $B(f, g)$ and $\Gamma_{f}(g):=\frac{p}{p-2}[A(f, g)-B(f, g)]$ belong to $R\left(P^{*}\right)$. Set

$$
Q_{f} g=\left\langle\left\langle u_{f}, g\right\rangle\right\rangle u_{f}
$$

We have then

$$
\begin{gathered}
M_{f}(g)=N(f)^{p-2}\left[2 g+(p-2) E_{f} g\right] \\
\Gamma_{f}(g)=p N(f)^{p-2} Q_{f} g
\end{gathered}
$$

Then $M_{f}$, resp. $\Gamma_{f}$ are bounded linear, resp. antilinear operators from $L_{p}(H)$ into $L_{p_{*}}(H)$, and $Q_{f}$ is a contractive antilinear endomorphism of $L_{p}(H)$ such that $Q_{f}^{2}=$ $E_{f}$; moreover:

$$
M_{f} P=P^{*} M_{f} P, \quad \Gamma_{f} P=P^{*} \Gamma_{f} P .
$$

Consider the positive symmetric bounded bilinear form defined on $L_{p}(H)$ by

$$
(g, h)_{f}:=\left\langle M_{f}(g), h\right\rangle=\int N(f)^{p-2}\left\langle\left\langle\left(2 I+(p-2) E_{f}\right) g \mid h\right\rangle\right\rangle d \mu
$$


Note that $\Gamma_{f}=M_{f} Q_{f}=Q_{J_{p}} M_{f}$ and $Q_{f}^{*}=Q_{J_{p} f}$; then $Q_{f}$ is hermitian for $(\cdot, \cdot)_{f}$ since

$$
\begin{aligned}
\left(Q_{f} g, h\right)_{f} & =\left\langle M_{f} Q_{f} g, h\right\rangle=\left\langle Q_{f} g, M_{f} h\right\rangle \\
& =\left\langle Q_{J_{p} f} M_{f} h, g\right\rangle=\left\langle M_{f} Q_{f} h, g\right\rangle=\left(Q_{f} h, g\right)_{f}
\end{aligned}
$$

On the other hand $P$ is hermitian for $(\cdot, \cdot)_{f}$ since (using $(21)$ )

$$
\begin{aligned}
(P g, h)_{f} & =\left\langle M_{f} P g, h\right\rangle=\left\langle P^{*} M_{f} P g, h\right\rangle=\left\langle M_{f} P g, P h\right\rangle=(P g, P h)_{f} \\
& =\overline{(P h, P g)_{f}}=\overline{(P h, g)_{f}}=(g, P h)_{f}
\end{aligned}
$$

Let $N_{f}$ be the kernel of the form $(\cdot, \cdot)_{f}$ : we have $g \in N_{f}$ iff $(g, g)_{f}=0$ iff $(g, h)_{f}=0$ for all $h \in L_{p}(H)$ (by Cauchy-Schwartz inequality). Then $P N_{f} \subset N_{f}$ since

$$
(P g, P g)_{f}=(g, P g)_{f}=0 \quad \text { if } g \in N_{f}
$$

On the other hand the operator $2 \cdot \mathbf{1}_{\mathbf{V S}(f)}+(p-2) E_{f}$ maps $L_{p}(H)$ onto $\mathbf{1}_{\mathbf{V S}(f)} L_{p}(H)$; hence $g \in N_{f}$ iff $\left\langle N(f)^{p-2} g, h\right\rangle=0$ for every $h \in 1_{\mathbf{V S}(f)} L_{p}(H)$ iff $N(f)^{p-2} g=0$ iff $\mathbf{1}_{\mathbf{V S}(f)} g=0$.

We have thus $R\left(F_{f}\right)=N_{f}$ and consequently

$$
P F_{f}=F_{f} P F_{f}
$$

Since $L_{p}(H)$ is a strictly convex Banach space as well as its dual, we have by the auxiliary Lemma 6.1 below:

$$
P F_{f}=F_{f} P
$$

Let us show that $Q_{f} P$ is hermitian for $(\cdot, \cdot)_{f}$, using eq. (21) again:

$$
\begin{aligned}
\left(Q_{f} P g, h\right)_{f} & =\left\langle M_{f} Q_{f} P g, h\right\rangle=\left\langle\Gamma_{f} P g, h\right\rangle \\
& =\left\langle P^{*} \Gamma_{f} P g, h\right\rangle=\left\langle\Gamma_{f} P g, P h\right\rangle \\
& =\left(Q_{f} P g, P h\right)_{f}=\left(Q_{f} P h, P g\right)_{f} \\
& =\left(Q_{f} P h, g\right)_{f}
\end{aligned}
$$

Since $Q_{f}$ and $P$ are separately hermitian for $(\cdot, \cdot)_{f}$ we have

$$
\left(Q_{f} P g, h\right)_{f}=\left(P Q_{f} h, g\right)_{f},
$$

hence $\left(P Q_{f}-Q_{f} P\right) h \in N_{f}$, i.e. $\left(I-F_{f}\right) P Q_{f}=\left(I-F_{f}\right) Q_{f} P$. Composing on the left by $G_{f}$ and on the right by $Q_{f}$, or conversely, we obtain

$$
G_{f} P E_{f}=0=E_{f} P G_{f} .
$$

Since, on the other hand,

$$
F_{f} P E_{f}=P F_{f} E_{f}=0=E_{f} F_{f} P=E_{f} P F_{f},
$$


we obtain

$$
P E_{f}=E_{f} P E_{f}=E_{f} P .
$$

We state now and give a proof of the announced auxiliary Lemma.

Lemma 6.1. Let $X$ be a strictly convex Banach space with strictly convex dual, and $P, Q$ two contractive projections on $X$. The following conditions are equivalent:

(i) $P Q$ is a projection.

(ii) $P Q=Q P Q$.

(iii) $P Q=P Q P$.

If moreover the complementary projection $Q^{\perp}$ is contractive too then $P Q=Q P$.

Proof. If (ii) is verified then $(P Q)^{2}=P Q P Q=P \cdot P Q=P Q$; while if (iii) is verified then $(P Q)^{2}=P Q P Q=P Q \cdot Q=P Q$. Hence both (ii) and (iii) imply (i) (without any contractiveness assumption). Conversely if (i) is verified then for every $x \in R(P Q)$ we have $x=Q x=P Q x$ (by [2, Prop. 1.1 (iii)]; only the strict convexity of $X$ is needed) so $x \in R(P) \cap R(Q)$. Since the converse is trivial, we see that $R(P Q)=R(P) \cap R(Q)$; in particular $Q P Q=P Q$ and (ii) is verified. Dualizing we have that $P^{*}, Q^{*}$ and $Q^{*} P^{*}$ are contractive projections in $X^{*}$; hence $Q^{*} P^{*}=P^{*} Q^{*} P^{*}$, so $P Q=P Q P$ and (iii) is verified. Now (iii) implies $P Q^{\perp}=P Q^{\perp} P$, and if $Q^{\perp}$ is contractive this implies $P Q^{\perp}=Q^{\perp} P Q^{\perp}$ by the preceding. Then

$$
Q=P Q+P Q^{\perp}=Q P Q+Q^{\perp} P Q^{\perp}
$$

which in turn implies $Q P=P Q=Q P Q$.

Remark. The final assertion $P Q=Q P$ of Lemma 6.1 is stated in [2] (for $X=C_{p}$ ) as Cor. 1.7 without the assumption that the complementary projection $Q^{\perp}$ is contractive. This statement is not correct: if $p \neq 2$ it is easy to construct rank 1 contractive projections $P, Q$ in $X=\ell_{p}$ or $C_{p}$ such that $P Q=0 \neq Q P$ : choose non zero elements $a, b \in X$ such that their norming functionals $J a, J b$ verify $\langle J a, b\rangle=0$ and $\langle J b, a\rangle \neq 0$ and set $P=a \otimes J a, Q=b \otimes J b$.

Acknowledgements. The author thanks warmly C. W. Henson for spending time in discussions on ultraroots and axiomatizability in Functional Analysis. 


\section{References}

[1] T. Andô, Contractive projections in $L_{p}$ spaces, Pacific J. Math. 17 (1966), 391-405.

[2] J. Arazy and Y. Friedman, Contractive projections in $C_{p}$, Mem. Amer. Math. Soc. 95 (1992).

[3] S. J. Bernau and H. E. Lacey, The range of a contractive projection on an $L_{p}$-space, Pacific J. Math. 53 (1974), 21-41.

[4] R. G. Douglas, Contractive projections on an $L_{1}$ space, Pacific J. Math. 15 (1965), 443-462.

[5] I. Doust, Contractive projections on Lebesgue-Bochner spaces, Function Spaces (Edwardsville, IL, 1994), Lecture Notes in Pure and Appl. Math., vol. 172, Dekker, New York, 1995, pp. 101109

[6] P. G. Dodds, C. B. Huijsmans, and B. de Pagter, Characterizations of conditional expectationtype operators, Pacific J. Math. 141 (1990), 55-77.

[7] D. H. Fremlin, Topological Riesz spaces and measure theory, Cambridge University Press, London, 1974 .

[8] S. Guerre and Y. Raynaud, Sur les isométries de $L^{p}(X)$ et le théorème ergodique vectoriel, Canad. J. Math. 40 (1988), 360-391.

[9] C. W. Henson and J. Iovino, Ultraproducts in analysis, Analysis and Logic (Mons, 1997) (C. Finet and C. Michaux, eds.), London Math. Soc. Lecture Note Ser., vol. 262, Cambridge Univ. Press, Cambridge, 2002, pp. 1-110.

[10] C. W. Henson and Y. Raynaud, On the theory of $L_{p}\left(L_{q}\right)$ Banach lattices, in preparation.

[11] H. E. Lacey, The isometric theory of classical Banach spaces, Springer-Verlag, New York, 1974.

[12] B. Lemmens and O. van Gaans, On one-complemented subspaces of Minkowski spaces with smooth Riesz norms, Eurandom report (2002), preprint.

[13] M. Levy and Y. Raynaud, Ultrapuissances de $L^{p}\left(L^{q}\right)$, Seminar on Functional Analysis, 1983/1984, Publ. Math. Univ. Paris VII, vol. 20, Univ. Paris VII, Paris, 1984, pp. 69-79.

[14] B. Randrianantoanina, 1-complemented subspaces of spaces with 1-unconditional bases, Canad. J. Math. 49 (1997), 1242-1264.

[15] _ N Norm-one projections in Banach spaces, Taiwanese J. Math. 5 (2001), 35-95.

[16] (2003), private communication.

[17] L. Tzafriri, Remarks on contractive projections in $L_{p}$-spaces, Israel J. Math. 7 (1969), 9-15. 